\title{
Modeling \& Simulations in Photoelectrochemical Water Oxidation: From Single Level to Multi-scale Modeling
}

\author{
Xueqing Zhang and Anja Bieberle-Hütter* \\ Photo-electrochemical Materials and Interfaces, Dutch Institute for Fundamental Energy Research
} (DIFFER), De Zaale 20, 5612 AJ, Eindhoven, the Netherlands

\begin{abstract}
This review summarizes recent developments, challenges and strategies in the field of modeling and simulations of photoelectrochemical (PEC) water oxidation. We focus on water splitting by metal oxide semiconductors and discuss topics such as theoretical calculations of light absorption, band gap / band edge, charge transport, and electrochemical reactions at the electrode-electrolyte interface. In particular, we review the mechanisms of the oxygen evolution reaction (OER), strategies to lower overpotential, and computational methods applied to PEC systems with particular focus on multiscale modeling. The current challenges in modeling PEC interfaces and their processes are summarized. At the end, we propose a new multiscale modeling approach to simulate the PEC interface under conditions most similar to experiments. This approach will contribute to identifying the limitations at PEC interfaces. Its generic nature allows applying it to a number of electrochemical systems. Keywords: water splitting, photo-electrochemistry, modeling \& simulations
\end{abstract}

\section{Introduction}

Photoelectrochemical (PEC) solar fuel conversion is seen as one of our long term strategies to achieve clean energy and environmental sustainability for society. ${ }^{1-9}$ Despite many years of research in PEC solar fuel conversion since the pioneer work of Fujishima and Honda in $1972,{ }^{9}$ there is still a significant gap between photo-to-fuel conversion efficiency and maximal theoretical efficiency. ${ }^{10,11}$ This indicates that the indepth understanding of some key aspects of the PEC solar energy conversion process are still not fully understood. ${ }^{12}$

In PEC solar fuel conversion, a medium is converted with the help of sunlight and PEC active materials into a fuel. In the simplest way, this is the production of hydrogen by splitting of water. Figure 1 shows the principle of operation of a PEC cell for water splitting under an alkaline environment. Electrons are excited with the help of light illumination from their ground state (valence band, VB) to the excited state (conduction band, CB), leaving behind a positively charged hole in the VB. Hence, an electron-hole pair is created. In an n-type semiconductor, the electrons travel to the counter electrode where they reduce water and hydrogen gas is formed $\left(4 \mathrm{H}_{2} \mathrm{O}+4 \mathrm{e}^{-} \rightarrow 4 \mathrm{OH}^{-}+2 \mathrm{H}_{2}\right)$. The holes migrate to the surface, where they oxidize water to form oxygen gas $\left(4 \mathrm{OH}^{-}+4 h^{+} \rightarrow 2 \mathrm{H}_{2} \mathrm{O}+\mathrm{O}_{2}\right) .{ }^{13}$ The charge transport is limited by the nature of the semiconductor, the electrochemical reactions are limited by the catalytic activity of the electrode material. Thus, both the semiconductor physics and the surface chemistry have to be carefully considered for optimization of PEC systems. ${ }^{12,14}$ Current research strongly focuses on the oxygen evolution reaction, because it accounts for most of the overpotential required to drive water splitting, and due to the four electron process which is more complicated than hydrogen evolution. 


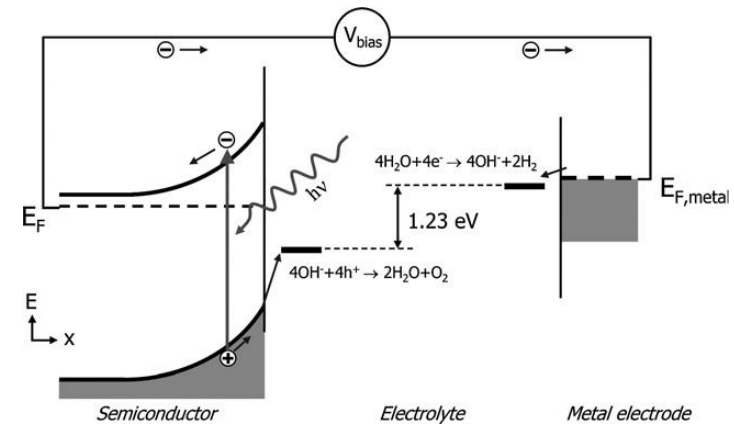

Figure 1. Schematic of photoelectrochemical (PEC) solar fuel conversion in an alkaline environment. ${ }^{13}$

A PEC system consists of several interfaces. Figure 2 shows a schematic sketch of a half cell of a PEC system. It consists of the electrolyte and the electrode; the electrode can be made of a metal or a semiconductor and might include a co-catalyst. There are three interfaces of which two are solid-liquid (semiconductorelectrolyte (IF1) and co-catalyst-electrolyte (IF2)) and one is solid-solid (semiconductor-co-catalyst (IF3)). Multiple processes, such as charge transport, charge transfer, adsorption/desorption, and electrochemical reactions, take place at these interfaces. The reaction mechanisms at the interfaces are complex and are not fully identified even for the most common systems. ${ }^{12,14}$

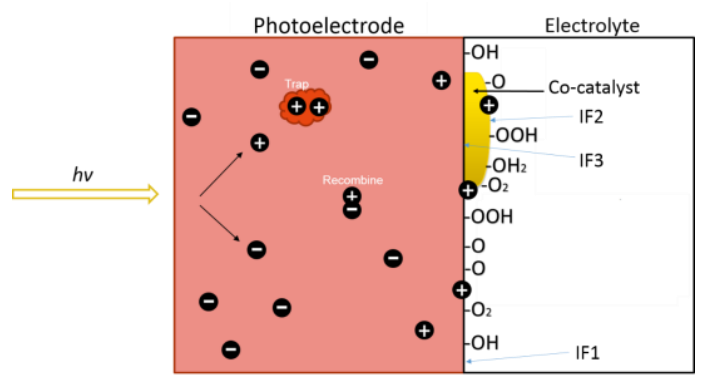

Figure 2. Sketch of a half cell of a PEC system. The interfaces are indicated by IF1 (semiconductor-electrolyte interface ), IF2 (co-catalyst-electrolyte interface), and IF3 (semiconductor-cocatalyst interface). $-\mathrm{OH},-\mathrm{O},-\mathrm{OOH},-\mathrm{OH}_{2}$, and $-\mathrm{O}_{2}$ are intermediate species at the interface.

Since there are multiple processes, multiple interfaces, and unclear reaction mechanisms at the interfaces, the interpretation of experimental electrochemical measurements is difficult. Recent advances in modeling and simulation techniques allow for computational design of such interfaces at the atomistic level. 12, 14, 15 A few excellent theoretical review articles focusing solely on first principle calculations of PEC interfaces have recently been published. ${ }^{12,14-16}$ Liao and Carter ${ }^{14}$ reviewed the various aspects of a photocatalytic cycle: light absorption, electron/hole transport, band edge alignments of semiconductors, and surface chemistry. Strategies to improve each aspect are discussed as well as shortcomings of current modeling and simulation efforts, such as simulating kinetic barriers of proton coupled electron transfer, direct simulations of chemical reactions in the presence of photo-generated electrons and holes, and consideration of the complex interactions between solid semiconductor and aqueous solution. Akimov et al. ${ }^{12}$ reviewed the theoretical methods that are used in this field, as well as the dynamics of charge separation, diffusion, relaxation, recombination, the chemical and electronic structures of photoactive materials at oxide interfaces, and also mechanisms of electron transfer processes. After discussion of theoretical methods and achievements in these aspects, the authors outlined future research directions. New theoretical frameworks and tools must be developed. The light-matter interactions and multiscale paradigms are promising and most required future research directions. Dau et al. reviewed water oxidation from various fields of research, i.e. heterogeneous, homogeneous and biological catalysts. ${ }^{17}$ They conclude that unifying concepts between the different fields are required not only on a theoretical, conceptual level but also in terms of new technological systems. A combined theoretical and experimental perspective of solar hydrogen production with semiconductor metal oxides was presented by a consortium around Valdes et al. ${ }^{15}$ In the theoretical part, the authors showed that computational studies of the OER in electrolysis have offered many useful insights, such as the universal applicability of the four step mechanism (as discussed in the text) to analyze the OER reaction on metal oxides and the photo-oxidation of water.

The above discussed reviews focus only on first principle calculations; they contribute strongly to the atomistic understanding of the PEC interface. However, a paper that reviews the PEC processes on a multiscale level is still missing and would allow bridging between 
modeling \& simulations and experimental electrochemical studies.

This review aims at filling this gap. It is structured as follows. In chapter 2, we discuss the theoretical contributions to specific aspects of the PEC process. Particularly, the various proposed OER mechanisms and the strategies to improve the overpotential are discussed. In chapter 3 , we discuss the computational methods that were applied for investigations of PEC interfaces with special focus on multiscale modeling. In chapter 4, we summarize the current challenges in modeling and simulations of PEC interfaces. To meet the current challenges, we propose a multiscale modeling approach for PEC systems in chapter 5 .

\section{Modeling \& Simulations of PEC processes}

Substantial contributions have been made by modeling and simulations to understanding and optimizing the PEC interface. ${ }^{12,14,15,18}$ In this section, we first shortly introduce the main theoretical methods. We focus then on the processes taking place at the PEC interface and discuss these with examples of computational studies from the literature.

\subsection{Theoretical methods}

In this subsection, we shortly introduce the theoretical methods that are relevant to this review. The application of these methods will be discussed later in the manuscript.

First of all, density functional theory (DFT) is a very popular computational method in many research fields. ${ }^{19-21}$ DFT is a first-principles tool that can be used to understand catalytic processes and identify promising candidate materials through the calculation of kinetic and thermodynamic properties. ${ }^{19}, 20$ Many modern computational methods were derived from DFT. In PEC solar fuel conversion, the first process is the excitation of the semiconductor induced by the light. Electronic excitations lie at the origin of most of the commonly measured spectra. However, the computation of excited states requires a larger effort than ground-state calculations. ${ }^{22,} 23$ The two main approached to calculate excitation energies (e.g., the optical spectrum) are (i) many-body perturbation theory (MBPT), is based on a set of Green's-function equations, starting with a one-electron propagator and considering the electron-hole Green's function for the response; (ii) time-dependent DFT (TD-DFT), which offers the important practical advantage of a dependence on density rather than on multivariable Green's functions. ${ }^{23}$

The dynamics of the molecular systems can be studied at DFT level by combining molecular dynamics (MD) and DFT, which is usually mentioned as $\mathbf{A b}$ initio molecular dynamics (AIMD). AIMD has been used in the field of realistic computer simulation of complex molecular systems and processes, including chemical reactions. ${ }^{24-26}$ One of the most popular AIMD methods is Car-Parrinello molecular dynamics (CPMD). ${ }^{26}$ The CPMD code is a parallelized plane wave / pseudopotential implementation of DFT, particularly designed for AIMD. ${ }^{27}$

Molecular dynamics (MD) simulation ${ }^{28}$ provides the methodology for detailed microscopic modeling on the molecular scale. The nature of matter is to be found in the structure and motion of its constituent building blocks, and the dynamics is contained in the solution to the $\mathrm{N}$-body problem. MD allows to follow the movement of individual atoms/molecules. One of the popular classical MD methods is the reactive force field molecular dynamics (ReaxFF MD), which is discussed in this review for its applications in water splitting. ReaxFF MD is a method for modeling chemical reactions with atomistic potentials based on the reactive force field approach developed by van Duin, Goddard and co-workers. ${ }^{29}$

Broadly speaking, kinetic Monte Carlo (kMC) can be applied to any system describable as a set of minima of a potential-energy surface, the evolution of which will then be regarded as hops from one minimum to a neighboring one. ${ }^{30}$ The hops in $\mathrm{kMC}$ are modeled as stochastic processes and the algorithms use random numbers to determine at which times the hops occur and to which neighboring minimum they go. ${ }^{30-32}$

State-space modelling (SSM) is known in control theory to simulate complex, interdepending systems. ${ }^{33}$ The state-space representation is a mathematical 
model of a physical system as a set of input, output and state variables related by first-order differential equations.

Traditionally, a computational method uses a single computational tool to model a given system at a particular time and length scale. Each level of theory focuses on the system under a single aspect.

Multiscale modeling aims at stitching multiple aspects together into a unified whole, such that macroscopic properties emerge from underlying microscopic phenomena. ${ }^{34}$ Multiscale modeling uses two or more models for different scales as shown in Figure 3.

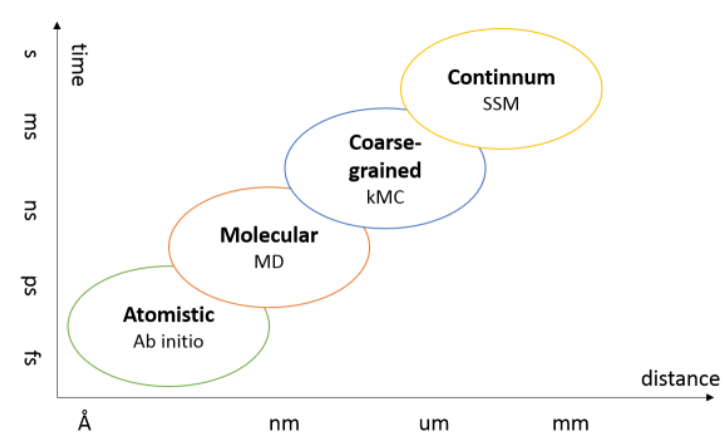

Figure 3. A schematic of the methods of multiscale modelling.

\subsection{Light absorption}

The first process of PEC water splitting is the absorption of sunlight by the photoelectrode. Thus, one of the challenges in the optimization of this process is to find efficient absorbers of sunlight. Ping et al. ${ }^{16}$ presented state-of-the-art methods for the calculation of electronic excitations in solids and molecules based on MBPT. The authors highlighted that solving the Bethe-Salpeter equation (BSE) ${ }^{35}$ based on MBPT and the Green's function formalism to obtain absorption spectra has been the strategy of choice for most solids. ${ }^{16}$ In the case of molecules many calculations of absorption spectra are carried out using TD-DFT. ${ }^{16}, 36$ The authors compared the absorption spectrum of bulk silicon computed by solving the BSE using the TD-DFT, with that of experimental results. ${ }^{16}$ The computed spectrum by BSE exhibits accurate position and intensity of the two main peaks, compared to experiments. The TDLDA calculations yield an absorption threshold much lower than found both experimentally and using the BSE as shown in Figure 4. In addition, the TDLDA spectrum exhibits a shoulder instead of a main peak. This example shows the advantage of BSE in calculation absorption spectra of bulk materials with respect of TDDFT.

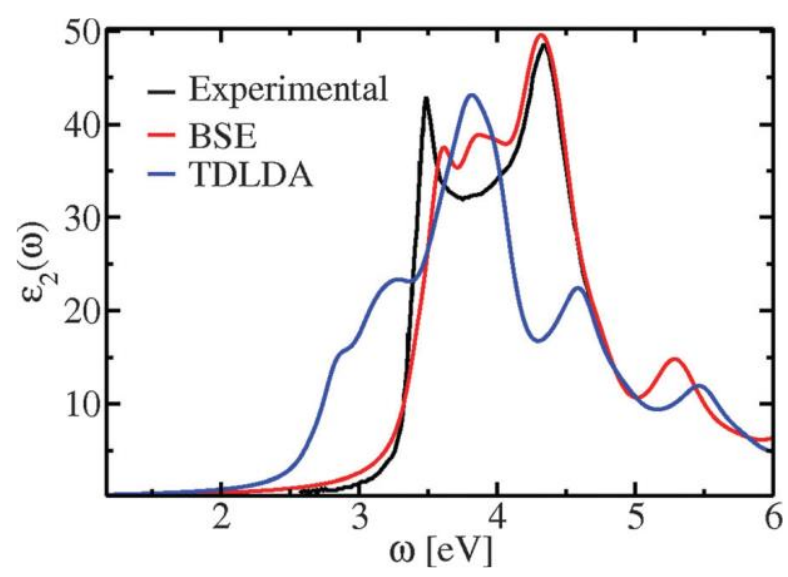

Figure 4. Absorption spectrum of bulk silicon computed by solving the BSE and with the TDLDA (TD-DFT with localdensity approximations), compared to experimental results. ${ }^{16}$, 37

Recently, Kazaryan et al. ${ }^{38}$ demonstrated the importance of excitation in calculation of light induced water splitting process. The authors studied water oxidation by $\mathrm{Ti}(\mathrm{OH})_{4}$ in the ground and excited states using density functional (Delta $\mathrm{SCF}^{39}$ and TD-DFT) methods. They found that the excitation is crucial for the $\mathrm{H}$-abstraction from water. This is primarily dictated by the energy needed for the liberation of $\mathrm{OH}$. In the ground state, even bound hydroxyl radicals are not being formed. On the contrary, excitation opens an alternative route that allows for $\mathrm{OH}$ radical generation. The overall energetics of the oxygen and hydrogen generation reactions (Figure 5) is dominated by the energy required for the hydroxyl radical generation. This energy is determined by the energies of the products $\mathrm{Ti}(\mathrm{OH})_{3} \mathrm{H}_{2} \mathrm{O}+\mathrm{OH}$ relative to the reactants $\mathrm{Ti}(\mathrm{OH})_{4}+$ $\mathrm{H}_{2} \mathrm{O}$. We can see from Figure 5 that each reaction of $\mathrm{H}$ abstraction driven by $\mathrm{Ti}(\mathrm{OH})_{4}$ can only proceed on the excited state surface that exhibits low to moderate activation barriers. ${ }^{38}$ 


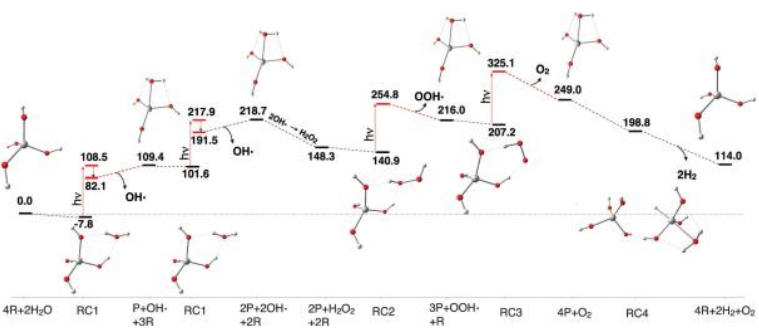

Figure 5 Relative ground (black bars) and excited (red bars) state potential energies of the main intermediates in the reactions. The vertical excitation is indicated by a vertical red arrow. The reactant $\mathrm{Ti}(\mathrm{OH})_{4}$ and product $\mathrm{Ti}(\mathrm{OH})_{3} \mathrm{H}_{2} \mathrm{O}$ compounds are labelled by $R$ and $P$, respectively.

The light absorption by semiconductor has been well described by MBPT. ${ }^{16}$ However, the simulation of photoexcitation as the driving force of the water splitting reaction has only been demonstrated for small mode systems. ${ }^{38,40}$ In most of the literature, as discussed below, water decomposition at the semiconductor surface is viewed as an electro-catalytic process driven by the electrochemical potential.

\subsection{Electron - hole separation and band gap/edge engineering}

An important value for the performance of a photoelectrode is the bandgap of the material, i.e., the energy difference between the VB and CB. The value of the band gap effectively determines the maximum energy available for the electrochemical reactions to take place. The free energy for water splitting dictates that the material must have a band gap of at least 1.23 eV. Due to thermodynamic losses and kinetic barriers, the favorable band gaps range between $2-2.5 \mathrm{eV} .{ }^{14}$ The band gaps of metal oxides usually range between 2 and $7 \mathrm{eV}$. Large bandgap materials are transparent for photons in the visible spectrum and hence, these materials are not desirable for photoelectrochemical application.

The band gap and the band edge positions can be tuned if they do not fit the PEC requirements. Among the most popular methods for photocatalytic applications are the use of dopants and the formation of alloys. It has been predicted theoretically that doping $\mathrm{WO}_{3}$ with a low valence ion such as $\mathrm{Hf}$ shifts the $\mathrm{CB}$ to energies high enough to drive the hydrogen evolution reaction (HER). ${ }^{41}$ Mono-doping usually has the shortcoming of limited reduction of the band gap. Based on band structure calculations, Gai et al. ${ }^{42}$ compared monodoping with co-doping. $\mathrm{TiO}_{2}$ was doped with n-type dopants ( $3 \mathrm{~d}$ transition metals: $\mathrm{V}, \mathrm{Cr} ; 4 \mathrm{~d}$ transition metals: $\mathrm{Nb}, \mathrm{Mo})$ on the Ti sites and p-type (C, N) dopants on the oxygen sites. The p-type dopants induces changes in the density of states (DOS) above the valence band maximum (VBM) of $\mathrm{TiO}_{2}$. The n-type dopants cause little perturbation at the conduction band minimum (CBM). It was found that co-doping of $n$ - and p-type dopants contributes more DOS to change the VBM and shifts the valence band edge up significantly (Figure 6). Thus, co-doping is demonstrated to be a promising way to modify the band gap and band edge positions.

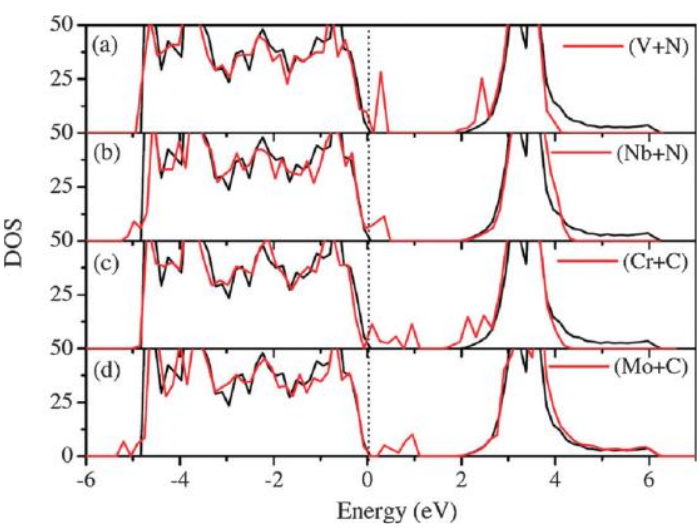

Figure 6. The Density of states (DOS) of undoped $\mathrm{TiO}_{2}$ (black) and $(\mathrm{V}+\mathrm{N}),(\mathrm{Nb}+\mathrm{N}),(\mathrm{Cr}+\mathrm{C})$, and $(\mathrm{Mo}+\mathrm{C})$-codoped $\mathrm{TiO}_{2}($ red $) .{ }^{42}$

In another study by Kanan and Carter, ${ }^{43} \mathrm{MnO}$ was alloyed with $\mathrm{ZnO}$. In this combined density functional theory and many-body Green's function theory scheme, the band gap of $\mathrm{MnO}$ was reduced by the alloying while maintaining the advantageous band edge positions (Figure 7). The 1:1 alloy of $\mathrm{MnO}$ and $\mathrm{ZnO}$ is identified as a new (2.6 eV band gap) visible-light-absorbing material with band edges suitably placed with respect to water-oxidation reactions. 


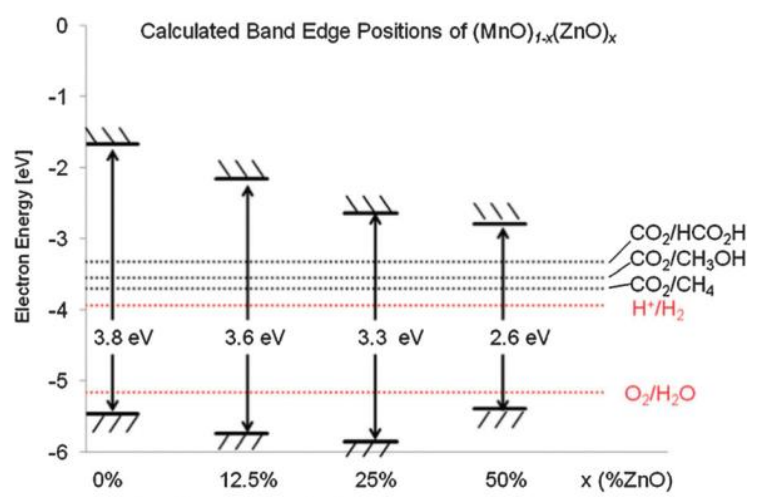

Figure 7. Predicted band edge positions (solid black lines) for $(\mathrm{MnO})_{1-\mathrm{x}}(\mathrm{ZnO})_{\times}$with different percentages of $\mathrm{ZnO} .^{43}$

Toroker et al. ${ }^{44}$ used hybrid density functional theory (DFT) and $\mathrm{DFT}+\mathrm{U}$ to investigate the electronic structure of binary oxide alloys. The band gaps of materials with gaps too large for efficient solar energy conversion, such as $\mathrm{MnO}, \mathrm{NiO}, \mathrm{ZnO}$, and $\mathrm{MgO}$, were reduced when alloyed with iron oxide (Figure 8). A range of band gaps for a variety of materials are obtained, where the alloys $\mathrm{Fe}_{1-\mathrm{x}} \mathrm{Ni}_{\mathrm{x}} \mathrm{O}$ and $\mathrm{Fe}_{1-\mathrm{x}} \mathrm{Zn}_{\mathrm{x}} \mathrm{O}$ exhibit much lower gaps than the gaps of $\mathrm{Fe}_{1-\mathrm{x}} \mathrm{Mn}_{\mathrm{x}} \mathrm{O}$ and $\mathrm{Fe}_{1-\mathrm{x}} \mathrm{Mg}_{\mathrm{x}} \mathrm{O}$. The former are closer to the optimal band gaps for efficient solar energy conversion. ${ }^{44}$ The key concept is that the alloy band gap may be substantially lower than the band gaps of either of the two parent materials when the band gap center positions of the pure materials are significantly different. By analysing the band edge positions the authors predicted that most of them cannot be used for the oxidation of water since their VBM lies above the free energy for oxidizing water. One exception is $\mathrm{Fe}_{0.25} \mathrm{Ni}_{0.75} \mathrm{O}$, with band edges that are adequate for water splitting.

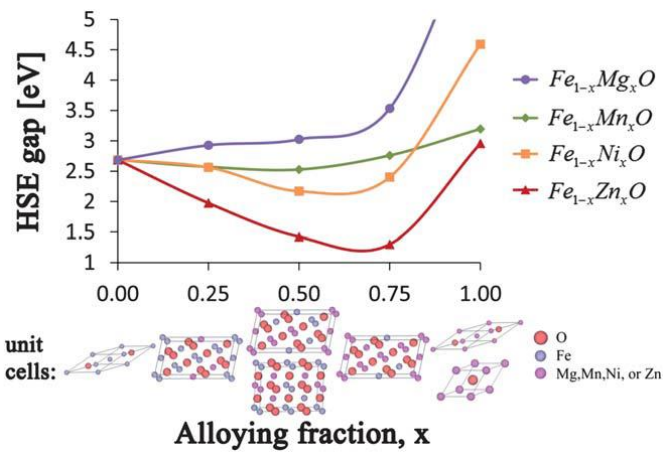

Figure 8. DFT evaluation of the band gap dependence on the alloying fraction $x$ for the alloys $\mathrm{Fe}_{1-x} \mathrm{Mg}_{\mathrm{x}} \mathrm{O}, \mathrm{Fe}_{1-x} \mathrm{Mn}_{\mathrm{x}} \mathrm{O}, \mathrm{Fe}_{1}$ ${ }_{x} \mathrm{Ni}_{x} \mathrm{O}$ and $\mathrm{Fe}_{1-x} \mathrm{Zn}_{\mathrm{x}} \mathrm{O}$. HSE is the Heyd, Scuseria, and Ernzerhof (HSE) hybrid exchange-correlation functional. ${ }^{44}$
The above discussed studies illustrate how recent theoretical studies can determine band gaps and how these can be tuned. Modeling and simulations allow here systematic studies in order to find most suitable compositions for water splitting. Experimental materials processing efforts can be considerably reduced due to such theoretical studies.

\subsection{Charge (electron / hole) transport}

Metal oxides are attractive candidates for PEC photoelectrodes owing to their low cost, earth abundance, and high stability under PEC conditions. However, their insulating nature limits the charge transport which is required for the carriers reaching the interface for electrochemical reactions to take place. ${ }^{45}$, 46 Possible strategies to improve the conductivity are doping $^{47}$ and nanostructuring. ${ }^{46}$ Modern nanotechnology allows the fabrication of very small structures which enable tunnelling as charge transport mechanism. ${ }^{46,48-50}$

There have been many contributions to the field of charge transport calculations. ${ }^{51-54}$ Here we discuss a few examples that are most relevant to PEC applications.

Liao and Carter et al. ${ }^{47}$ studied the electron transport in pure and doped hematite. Ab initio quantum mechanics was used to understand how titanium, zirconium, silicon, or germanium n-type doping affects the electron transport mechanism in hematite. They found that zirconium, silicon, or germanium doping is superior to titanium doping concerning charge transport because the former dopants do not act as electron trapping sites due to the higher instability of $\mathrm{Zr}$ (III) compared to $\mathrm{Ti}$ (III) and the more covalent interactions between silicon (germanium) and oxygen. ${ }^{47}$ Similarly, a DFT study of hole transport in nonstoichiometric and doped wustite revealed that vacancies create stronger traps than dopants. Copper and nitrogen dopants form deeper traps than lithium, sodium, or hydrogen. ${ }^{55}$

Besides doping another important strategy to enhance the conductivity of semiconducting materials is nanostructuring which allows for tunneling as a possible 
charge transport mechanism. Viswanathan and Norskov et al. ${ }^{46}$ presented a modelling study of the $\mathrm{TiO}_{2}$ electrochemical system using a metal-insulator-metal (MIM) configuration. The charge transport calculations are performed using density functional theory along with a Hubbard-U correction. The extracted kinetic current density is shown as a function of thickness at a fixed potential $U_{\mathrm{RHE}}=1.2 \mathrm{~V}$ (reversible hydrogen electrode) in Figure 9. Experimental data on ALD (atomic layer deposition) thin films measured by rotating disk electrode experiments are illustrated as well. The electrochemical current is normalized by the current at a thickness $2.1 \mathrm{~nm}$. A rapid decay in electronic conductivity with increasing thickness of the $\mathrm{TiO}_{2}$ layer is found. Below a limiting thickness the fixed potential $\left(\mathrm{U}_{\mathrm{RHE}}=1.2 \mathrm{~V}\right)$ is sufficient to drive the required electrochemical current (j/j0 with j0 the exchange current density). However, beyond the limiting thickness, there is a fast decrease in current. The critical thickness for tunneling is around $4 \mathrm{~nm}$. The finding of a critical thickness beyond which charge transport significantly affects the electrochemistry offers a guideline for nanostructuring requirements to enable traditionally insulating materials as thin film electrocatalysts.

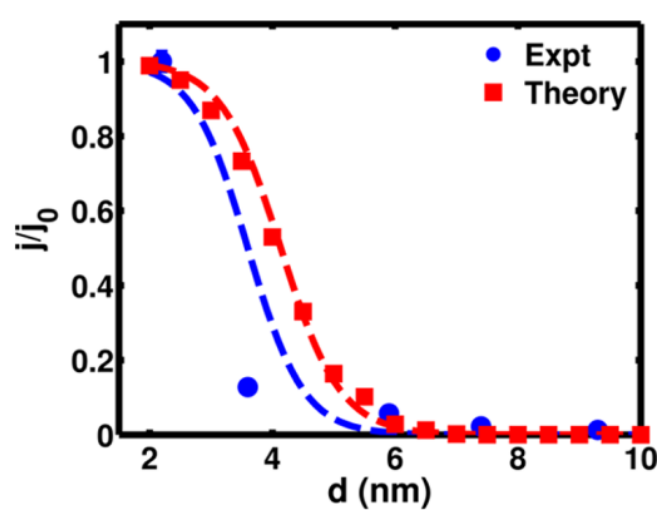

Figure 9. Theoretical and experimental plot of the extracted $\mathrm{j} / \mathrm{j} 0$ of the redox couple as a function of $\mathrm{TiO}_{2}$ thickness at a fixed potential. The experimental data points are from rotating disk electrode experiments carried out at 1600 rpm. ${ }^{46}$ The dashed lines are best-fit lines for the data points.

Achievements in computational studies have provided strategies for improving electron / hole transport properties of photoelectrodes. ${ }^{45-47}$ With an optimized thickness, one of the important strategies is to improve electron / hole transport by using dopants that increase carrier concentration without forming traps. ${ }^{47}$

\subsection{Electrochemical reactions}

Electrochemical reactions producing $\mathrm{H}_{2}$ or $\mathrm{O}_{2}$ at the electrode-electrolyte interface are another important step of the PEC process. The hydrogen evolution reaction is a two-electron process that usually proceeds with small overpotentials and is therefore not considered in this review. The investigation of the oxygen evolution reaction (OER), however, is much more challenging as it is a four-electron process that accounts for most of the overpotential required to drive water splitting. Reducing the OER overpotential lies in understanding the underlying reaction mechanism so that the rate-limiting step can be identified.

\subsubsection{OER mechanisms}

The oxygen evolution reaction is often regarded as a key reaction in developing strategies for renewable fuel synthesis from water. ${ }^{56}$ The mechanism of water oxidation depends on many effects, such as the electrode material, the potential applied, the surface termination and orientation of the photoelectrode (and co-catalyst). ${ }^{12,57-61}$ Under acidic conditions, the water oxidation reaction is $2 \mathrm{H}_{2} \mathrm{O}+4 \mathrm{~h}^{+} \rightarrow \mathrm{O}_{2}+4 \mathrm{H}^{+}$. Under alkaline conditions, it is $4 \mathrm{OH}^{-}+4 \mathrm{~h}^{+} \rightarrow \mathrm{O}_{2}+2 \mathrm{H}_{2} \mathrm{O}$. Thus, we distinguish in the following the acidic and alkaline OER mechanisms by the reactant species, e.g., $\mathrm{H}_{2} \mathrm{O}$ (for acidic) and $\mathrm{OH}^{-}$(for alkaline).

\subsubsection{OER in acidic medium}

The OER on a $\mathrm{TiO}_{2}$ photocatalyst has been extensively studied. ${ }^{62-64}$ In the 1980 s, Wilson et al. reported the formation of a surface state as a possible intermediate of the oxygen photoevolution reaction on $\mathrm{n}-\mathrm{TiO}_{2}$, as detected by a negative potential sweep after UV irradiation under anodic bias. ${ }^{65} \mathrm{~A}$ few years later, Salvador et al. claimed that Wilson's surface species may be attributed to adsorbed $\mathrm{H}_{2} \mathrm{O}_{2}$, produced by the coupling of surface $\mathrm{OH}$ radicals. ${ }^{66}$ The mechanism was updated in the 2000s. ${ }^{63,67}$ Using in-situ infrared (IR) adsorbed spectroscopy, Nakamura et al. claimed the 
occurrence of surface $\mathrm{OO}$ and $\mathrm{OOH}$ species. ${ }^{63,67}$ They proposed a mechanism of nucleophilic attack combined with oxidation that is intermediated by holes, followed by the coupling of $-\mathrm{OH}$ groups adsorbed on the surface $\left(\mathrm{TiO}_{2}\right) .{ }^{63,67}$ On the basis of their experimental data, ${ }^{63}$, 67 the authors proposed the following reaction mechanism (Figure 10) where $S$ is the active site at the surface.

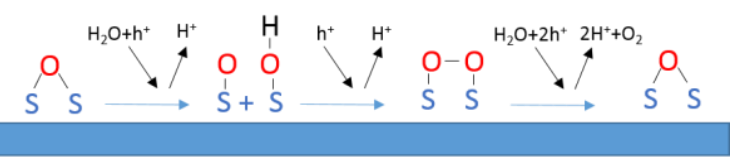

Figure 10. Sketch of OER mechanism according to Nakamura. ${ }^{63,67}$

By contrast, Rossmeisl and Norskov et al. ${ }^{57-59}$ proposed an OER mechanism, which considers a process where oxygen molecules are formed by an associative mechanism on the anode via a surface $\mathrm{HOO}^{*}$ intermediate. Direct recombination of oxygen atoms to form $\mathrm{O}_{2}$ was excluded because a large activation barrier is expected for this process. ${ }^{68}$ The theoretical model assumes a proton coupled electron transfer (PCET) process. This keeps the system neutral and avoids complexity of the charged system. It has become the widely used OER mechanism (Figure 11) under acidic condition, ${ }^{59}$

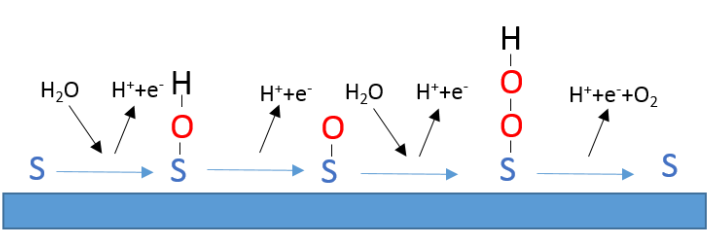

Figure 11. Sketch of OER mechanism according to Rossmeisl and Norskov. ${ }^{59}$

The mechanism for the electrochemical OER $\left(\mathrm{RuO}_{2}\right.$, $\mathrm{IrO}_{2}, \mathrm{WO}_{3}$ and $\mathrm{TiO}_{2}$ etc.) ${ }^{57-59}$ consists of four protoncoupled electron transfer steps as shown in the scheme above. This also indicates that to drive the process, each step must be supplied with a sufficient amount of energy, i.e. an applied voltage or solar energy is required. This external energy is modelled empirically by including the energy term $\mathrm{qU}$ in free energy calculations. This means that the potential affects the relative free energy through the chemical potential of the electrons (qU) in the electrode. This has been shown to predict trends for the OER quite well. ${ }^{57-59}$

By exploring the possible reaction channels and computing the Tafel lines, Liu et al. elucidated the OER kinetics on $\mathrm{RuO}_{2}(110) .{ }^{69}$ Above $1.58 \mathrm{~V}$, the reaction occurs on the fully $O$ terminated phase. The ratedetermining step is the water dissociation over two oxygens, which leads to the concurrent $\mathrm{O}-\mathrm{OH}$ bond formation. Figure 12 shows the free energy profiles and the molecular structures. Below $1.58 \mathrm{~V}$, the reaction occurs on a $\mathrm{OH} / \mathrm{O}$ mixed phase. The lowest energy pathway below $1.58 \mathrm{~V}$ involves the conversion of the $\mathrm{OH} / \mathrm{O}$ mixed phase to the O-terminated phase locally. The subsequent OER steps are exactly the same as those on the O-terminated phase above $1.58 \mathrm{~V}$.

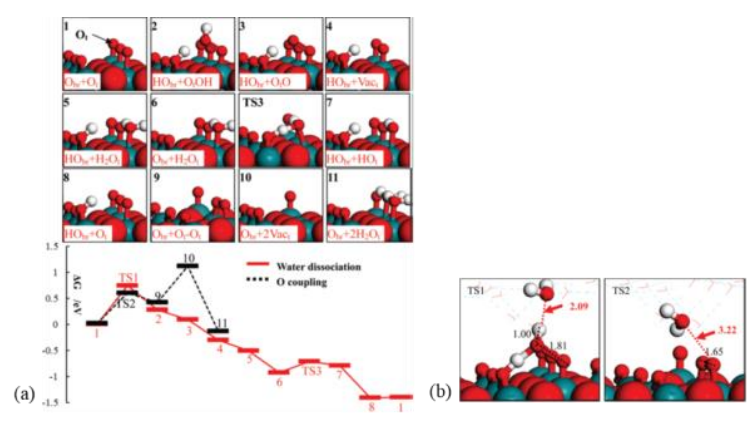

Figure 12. (a) The optimized structures of intermediate states and the free energy profile for OER on the O-terminated phase of $\mathrm{RuO} 2(110)$ at $1.58 \mathrm{~V}$. Obr, Ot, and Vact are the bridging O, the terminal O on Ru5c (five-coordinated) and the vacant Ru5c site, respectively. For the optimized structures, the first $\mathrm{H}_{2} \mathrm{O}$ layer is omitted for clarity. O: Red ball; $\mathrm{H}$ : white ball; Ru: Green ball. (b) Transition state structures of the water dissociation (TS1) and surface oxygen coupling (TS2) on the O-terminated phase of RuO2(110). All of the distances labelled are in Angstrom. ${ }^{69}$

\subsubsection{OER in alkaline medium}

Bockris and Otagawa ${ }^{70}$ provided a summary of five proposed pathways for the OER in the 1980s. These include Bockris's oxide path, Bockris's electrochemical path, Krasil'shchikov's path, O'Grady's path, and Kobussen's path. Sketches for each path are shown in Figure 13 derived from the respective literature. 
(a) Sketch of the Bockris's oxide path ${ }^{70}$ :

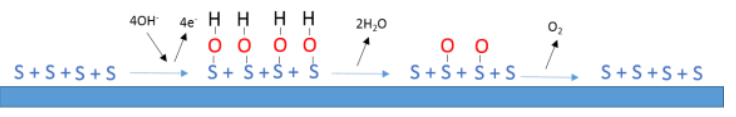

(b) Sketch of the Bockris's electrochemical path ${ }^{70}$ :

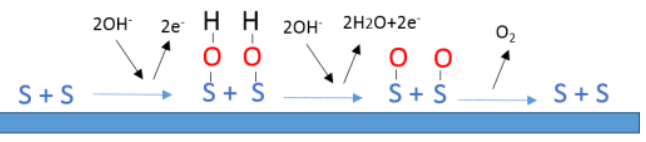

(c) Sketch of the Krasil'shchikov's path ${ }^{71}$ :

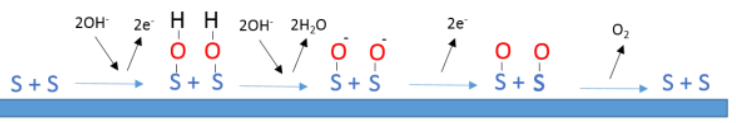

(d) Sketch of the O'Grady's path ${ }^{72}$ where, $S$ is a transition-metal ion with the valence state $z+$ at the surface of a perovskite:

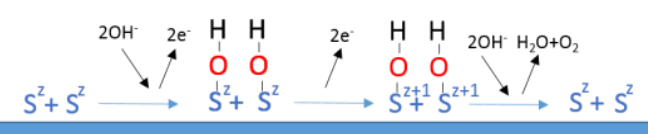

(e) Sketch of the Kobussen's path ${ }^{73}$ :

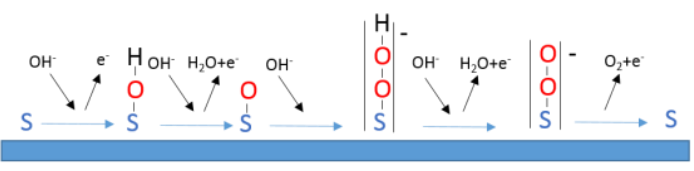

Figure 13. Sketchs of the OER mechanisms in alkaline medium: a) Bockris's oxide path ${ }^{70}$, b) Bockris's electrochemical path ${ }^{70}$, c) Krasil'shchikov's path ${ }^{71}$, d) O'Grady's path ${ }^{72}$, e) Kobussen's path. ${ }^{73}$.

The S-O formation was found to be the rate determining step in both Bockris's electrochemical path and O'Grady's path. Bockris's oxide path, Bockris's electrochemical path, Krasil'shchikov's path, O'Grady's path have the common $\mathrm{O}-\mathrm{O}$ bond formation through two adjacent intermediate species, S-O (or $\mathrm{S}-\mathrm{OH}$ ). Krasil'shchikov path was used to derive the OER mechanisms for passive oxide covered transition metal (nickel and cobalt) electrodes in aqueous alkaline solution. ${ }^{74,}{ }^{75}$ However, computational studies demonstrated that the direct recombination of oxygen atoms to form $\mathrm{O}_{2}$ has a large activation barrier. ${ }^{68}$

Direct recombination of oxygen atoms is excluded in the Kobussen's path. The surface intermediate species are, $-\mathrm{OH},-\mathrm{O}$ and $-\mathrm{OOH}$. The formation of hydrogen peroxide, $-\mathrm{OOH}$, has been assumed as rate determining in this mechanism to account for the kinetic parameters of oxygen evolution on $\mathrm{La}_{0.5} \mathrm{Ba}_{0.5} \mathrm{CoO}_{3}$. Both the intermediate species and the rate determining step are in agreement with modern computational studies. ${ }^{57-59}$

The above mentioned mechanisms were further studied in recent years. Now, one of the most widely used mechanism (mostly in theoretical works) of water oxidation under alkaline conditions is schematically shown in Figure $14 .^{76}$

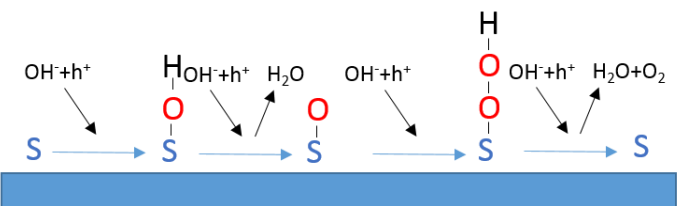

Figure 14. Sketch of the most widely used OER mechanism under alkaline conditions. ${ }^{76}$

This mechanism is very close to the one proposed by Kobussen et al. ${ }^{73}$. It is unique with respect to the formation of an $\mathrm{O}-\mathrm{O}-\mathrm{H}$ intermediate species.

Recently, Formal and co-workers ${ }^{56}$ presented an experimental study with the first rate law analysis of photo-induced water oxidation on a photo-to-anode surface. Using photo-induced absorption (PIA) spectroscopy and step on/off photo current measurements, they demonstrated the possibility of multi-hole concerted reactions on a hematite $\left(\mathrm{Fe}_{2} \mathrm{O}_{3}\right)$ surface. The authors focus specifically on how the reaction rate depends quantitatively on accumulation of photo-generated holes on the oxide surface. Two possible mechanisms of water oxidation on a hematite surface at high $\mathrm{pH}$ are suggested based on the experimental results (Figure 15). 


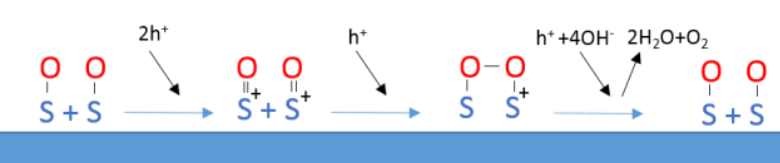

(a) mechanism A

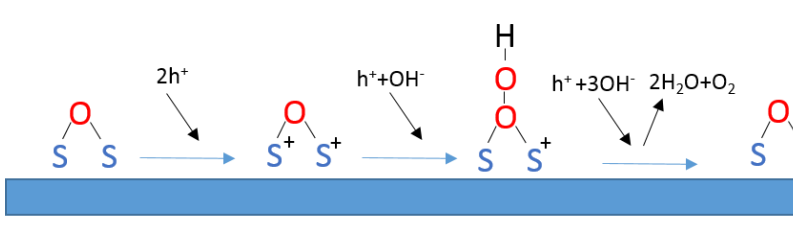

(b) mechanism B

Figure 15. Sketches of possible OER mechanisms on $\mathrm{Fe}_{2} \mathrm{O}_{3}$ at high $\mathrm{pH}$ according to Le Formal et al. $\mathrm{s}^{56}$ : a) mechanism $\mathrm{A}, \mathrm{b}$ ) mechanism $B$.

Mechanism A involves the coupling of two adjacent high-valent iron-oxo species on the surface, which may involve the transient formation of an $\mathrm{Fe}(\mathrm{V})$-oxo. Mechanism B proceeds via a nucleophilic attack of a hydroxide anion on a bridging surface oxygen, resulting in peroxide formation. It is stated that both mechanisms are possible; no selection criteria for one or the other mechanism is given in the paper. For either mechanism, the rate-determining step is the third oxidation and the resultant oxygen-oxygen bond formation. The O-O bond formation is found to be the rate determining step for both mechanisms, which is in agreement with theoretical predictions. ${ }^{44,57,60}$

In most of the theoretical modeling studies the electron transfer and proton transfer were assumed to occur simultaneously. To investigate more details of the PCET (proton coupled electron transfer) process, Chen and Selloni et al. presented a DFT study of the chemical dynamics of the first PCET on a $\mathrm{TiO}_{2}$ surface. Explicit water molecules were included in the modelling. The results suggest that the first PCET is sequential with the electron transfer following the proton transfer. ${ }^{62}$ On the basis of the analysis of the kinetics of the first PCET step on $\mathrm{TiO}_{2}$ the authors proposed the $\mathrm{pH}$ dependant mechanism. At low $\mathrm{pH}$ ( $\mathrm{pH}<$ point of zero charge), $\mathrm{OH}-$ groups are scarce on the $\mathrm{TiO}_{2}$ surface. Thus, proton transfer is rate determining with a moderate barrier. At higher $\mathrm{pH}\left(\mathrm{pH}>\right.$ point of zero charge), the $\mathrm{TiO}_{2}$ surface is covered by hydroxyl anions, and the overall PCET reaction rate does not depend on the $\mathrm{PT}$ (proton transfer) barrier. In this case, the PCET kinetics are determined by the barrier for ET (electron transfer), which is significantly smaller than that of PT. As a result, the OER is faster at high $\mathrm{pH}$.

In a recent paper, Zhou et al. studied the water oxidation mechanism on layered- birnessite-like manganese oxides. ${ }^{77}$ The authors proposed an oxidation mechanism similar to that of photosystem II (PSII), the first protein complex in the light-dependent reactions of oxygenic photosynthesis. ${ }^{78}$ The essential finding is that the $\mathrm{O}-\mathrm{O}$ bond formed between an $\mathrm{OH}$ - and a $\mu$-oxo. This is consistent with suggestions of Dau et al. that efficient water oxidation generally requires $\mu$-oxo bridging between metal ions. ${ }^{17}$

Figure 16 shows four routes of $\mathrm{O}-\mathrm{O}$ bond formation mechanisms formulated by Dau et al. ${ }^{17}$ Route $A$ involves partial water oxidation by peroxide formation at an early stage, that is, after accumulation of three oxidizing equivalents in the S3 state. This route is of high relevance in electrochemical water oxidation. ${ }^{57,59}$ In route $\mathrm{B}$, water oxidation is facilitated by a regular pattern of water-deprotonation steps, eventually resulting in two oxides terminally coordinated to two high-valent metal ions. The oxo group may have radical character and thus might be better described as an oxyl radical. Route $B$ is appealingly straightforward and has been invoked frequently in mechanistic models of water oxidation at electrode surfaces by metal complexes in solution (homogeneous catalysis) and also in PSII. ${ }^{17}$ In route $\mathrm{C}$, water oxidation involves bound or free hydroxides that act as proton acceptors in the O-O bond formation step. It relates to the 'alkaline mechanism' in the older electrochemical literature, in contrast to the 'acidic mechanism' of Route B, and typically is not considered a relevant pathway in the context of photosynthetic water oxidation. In route $D$, accumulation of oxidizing equivalents is not coupled to substrate-water deprotonation. Instead the catalyst is deprotonated, for example, by deprotonation of bridging oxides. Thereby 'acceptor bases' are created, which facilitate a direct coupling of water oxidation and water deprotonation in the O-O bond formation step. ${ }^{17}$ 


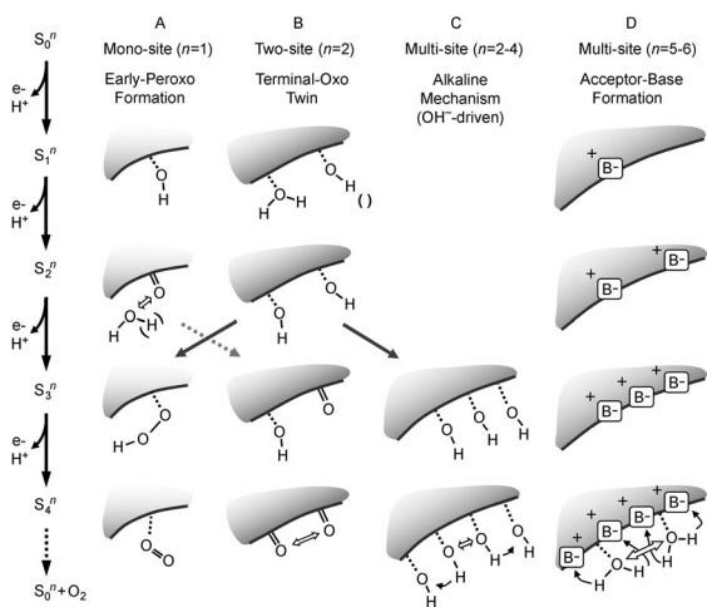

Figure 16. Four routes towards water oxidation. The catalyst is symbolized by the grey shape. In $\mathrm{Si}$, the number of accumulated oxidizing equivalents always is equal to i. However, accumulation of oxidizing equivalents (by oxidation of metal ions) is explicitly indicated only in $D$ in the form of the encircled ' + ' symbols. A-D differ in the minimal number of water and proton binding sites (n)..$^{17}$

In summary, until now the rate limiting steps of the OER have been studied for many materials at different conditions. The rate determining step varies depending on chemical conditions and the nature of the catalyst. ${ }^{57}$, 79-82 In most cases, the first or second deprotonation step is considered as rate limiting in the OER. Formation of the $\mathrm{O}-\mathrm{O}$ bond is also believed to be the rate limiting step for many materials. ${ }^{14,44,77}$ Roudgar et al. found that the removal of oxygen is an energetically strongly hindered step of the OER on a Pt cluster. ${ }^{80}$

\subsubsection{Strategies to reduce the OER overpotential}

The calculation of the thermodynamic overpotential has been popularized by Nørskov et al. ${ }^{83}$ and has made a considerable contribution to computational design of electrochemical processes. ${ }^{15,44,59,84-86}$ It also provides a simple yet powerful theoretical method for estimating reaction overpotentials using DFT calculations.

The OER overpotential $\eta$ is defined as

$\eta=\max \left[\Delta \mathrm{G}_{1}, \Delta \mathrm{G}_{2}, \Delta \mathrm{G}_{3}, \Delta \mathrm{G}_{4}\right] / \mathrm{e}-1.23[\mathrm{~V}]$,

where $\Delta G_{n}$ are the Gibbs free energy differences between PCET steps and $\mathrm{n}$ is the number of reactions. ${ }^{59}$

The OER overpotential can be changed by e.g. doping, mixed compound formation, addition of a co-catalyst, surface orientation, and the properties of the solvent. ${ }^{60}$, 85, 87, 8889 Here we discuss a few examples.
Liu et al. ${ }^{89}$ carried out DFT calculations in pure and doped $\mathrm{TiO}_{2}$. The Gibbs free energies of the first and second deprotonation step for different doping and surface terminations illustrate that the overpotential can be considerably decreased by $\mathrm{Mo}+\mathrm{C}$ doping (Table 1) and by changing the surface orientation due to a decrease of $\Delta \mathrm{G}_{\mathrm{i}}$.

Table 1. Comparison of the Free Energies of the First $(\Delta \mathrm{G} 1)$ and the Second $(\Delta G 2)$ Proton Removal Steps on the CoDoped $(\mathrm{Nb}+\mathrm{N}, \mathrm{Mo}+\mathrm{C})$ and Pure Anatase (101) and (001) Surfaces. ${ }^{89}$

$$
\mathrm{Nb}+\mathrm{N} \quad \mathrm{Mo}+\mathrm{C} \quad \text { pure }
$$

\begin{tabular}{|c|c|c|c|}
\hline \multicolumn{4}{|c|}{ (101) surface } \\
\hline$\Delta \mathrm{G} 1(\mathrm{eV})$ & 0.48 & 0.08 & 0.69 \\
\hline$\Delta \mathrm{G} 2(\mathrm{eV})$ & -0.80 & -0.39 & -0.96 \\
\hline \multicolumn{4}{|c|}{ (001) surface } \\
\hline$\Delta \mathrm{G} 1(\mathrm{eV})$ & 0.45 & 0.20 & 0.61 \\
\hline$\Delta \mathrm{G} 2(\mathrm{eV})$ & -1.08 & -0.82 & -1.20 \\
\hline
\end{tabular}

Friebel et al. ${ }^{85}$ found a 500 -fold enhancement of the OER activity (measured current density) of mixed ( $\mathrm{Ni}, \mathrm{Fe}$ ) oxyhydroxides $\left(\mathrm{Ni}_{1-\mathrm{x}} \mathrm{Fe} \mathrm{e}_{\mathrm{O}} \mathrm{OOH}\right)$ compared to the pure $\mathrm{Ni}$ and Fe parent compounds resulting in one of the most active currently known OER catalysts in an alkaline electrolyte. Operando X-ray absorption spectroscopy (XAS) using high energy resolution fluorescence detection (HERFD) reveals that $\mathrm{Fe}^{3+}$ in $\mathrm{Ni}_{1-x} \mathrm{Fe}_{\mathrm{x}} \mathrm{OOH}$ occupies octahedral sites with unusually short $\mathrm{Fe}-\mathrm{O}$ bond distances, induced by edge-sharing with surrounding $\left[\mathrm{NiO}_{6}\right]$ octahedra. DFT calculations demonstrated that this structure results in near optimal adsorption energies of OER intermediates and low overpotentials at Fe sites. By contrast, $\mathrm{Ni}$ sites in $\mathrm{Ni}_{1}$ ${ }_{x} \mathrm{Fe}_{\mathrm{x}} \mathrm{OOH}$ are not active sites for the oxidation of water.

Szyja and van Santen ${ }^{87}$ illustrated, by using AIMD, how the overpotential can be decreased by addition of a cocatalyst. They studied the $\mathrm{TiO}_{2}$ system with Co-oxide co-catalyst; the water molecules of the electrolyte are modelled explicitly. The essential finding is the observation of O-O bond formation at the interface of the $\mathrm{Co}$ oxide particle and $\mathrm{TiO}_{2}$ support. The synergetic effect leads to a low overpotential of only $0.32 \mathrm{eV} .{ }^{87}$ This value is lower than the one calculated $(0.48 \mathrm{~V})$ for the $\beta$ $\mathrm{CoOOH}$ phase ${ }^{90,91}$ and is very close to experimental observations of Khnayzer at al. ${ }^{92}$ for the cobalt 
phosphate cluster supported on titania, where in low $\mathrm{pH}$ the overpotential has been determined to be $0.37 \mathrm{~V}$.

Another excellent example of lowering the overpotential by a co-catalyst was given by Rossmeisl et al. ${ }^{93}$ The activity for the OER was enhanced by the interaction of gold with manganese and cobalt oxides. ${ }^{93}$ The activity was enhanced due to facilitated hydrogen transfer from ${ }^{*} \mathrm{OOH}$ to an adjacent acceptor site. The hydrogen transfer could occur either to an $\mathrm{Au}=\mathrm{O}$ acceptor site at an adjacent nanoparticle or, assuming the possibility of incorporating $\mathrm{Au}$ into the surface, to a Mn-O-Au site. The illustrative model is shown in Figure 17. The hydrogen transfer to a nearby Au nanoparticle is shown at the top and the incorporated Au site at the bottom. In the first pathway (upper highlight), the hydrogen transfer is facilitated by an adjacent Au nanoparticle. In the second pathway (lower highlight), the Mn-O-Au site functions as hydrogen acceptor, requiring $\mathrm{Au}$ to be incorporated into the $\mathrm{MnO}_{2}$.

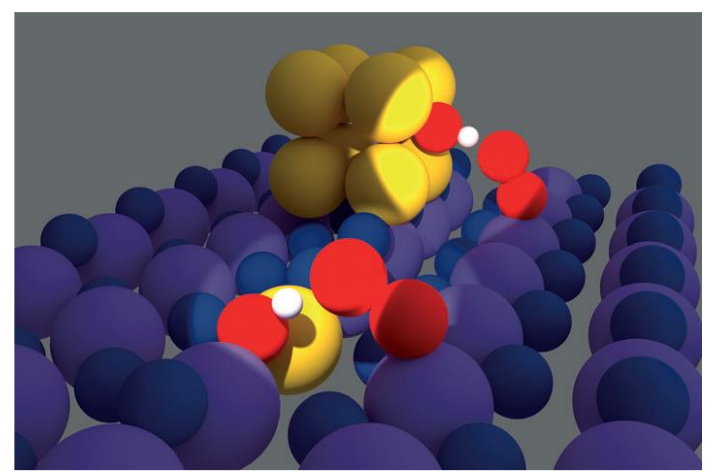

Figure 17. Model showing two different pathways for hydrogen transfer during the OER on a rutile (110) $\mathrm{MnO}_{2}$ surface. yellow: $\mathrm{Au}$, purple: $\mathrm{Mn}$, blue: lattice $\mathrm{O}$, red: reacting $\mathrm{O}$, and white: $\mathrm{H}$ atoms. ${ }^{93}$

Pronounced differences in free energy between diagrams with and without the hydrogen transfer mechanism are shown in Figure 18. The hydrogen transfer mechanism clearly reduces the energy required by the rate determining step, the step of O-O bond formation. There is a clear stabilization of the $\mathrm{Mn}=\mathrm{OOH}$ binding (Figure 18(b)), the energy of which becomes 3.5 $\mathrm{eV}$. At this point, only $0.3 \mathrm{eV}$ is required to facilitate $\mathrm{O}$ $\mathrm{O}$ bond formation. Correspondingly, the oxidation of water to a hydroxide becomes potential-determining, resulting in a decrease in the overall overpotential to only $0.4 \mathrm{~V}$.

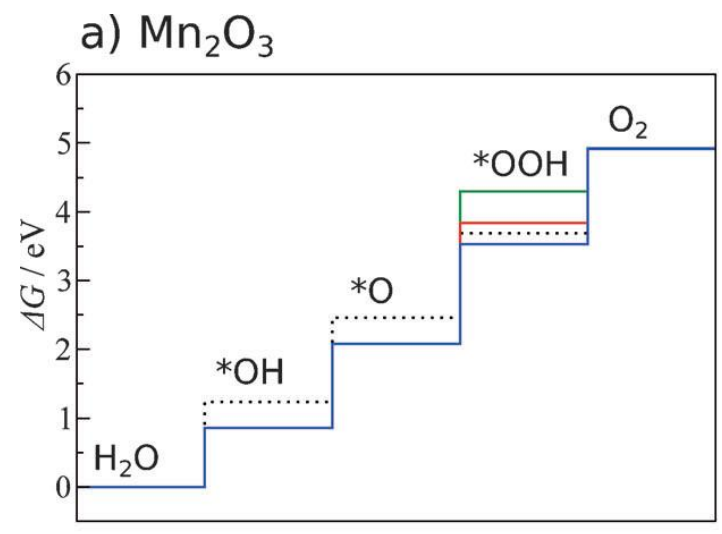

b) $\mathrm{MnO}_{2}$

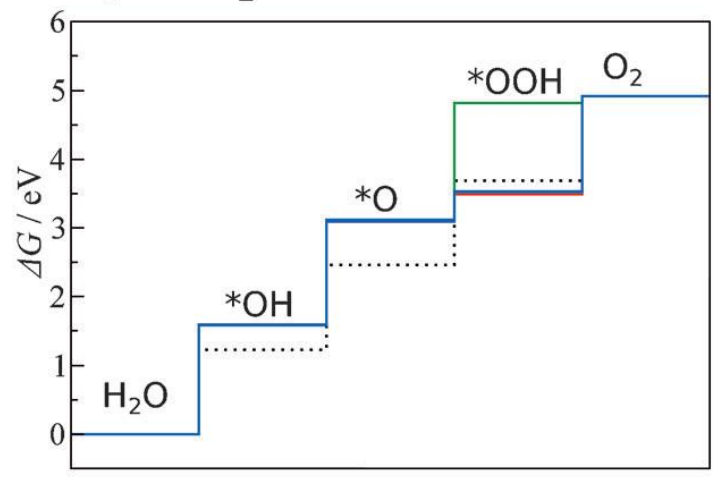

c) $\mathrm{CoO}_{x}$

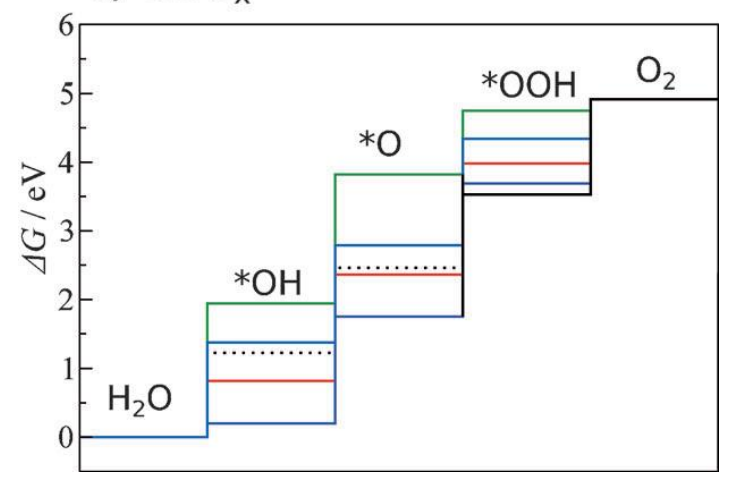

Figure 18. Free energy diagrams for the OER at zero applied potential. a) $\mathrm{Mn}_{2} \mathrm{O}_{3}$ without $\mathrm{H}$ transfer (green), with $\mathrm{H}$ transfer (red), and with $\mathrm{H}$ transfer to an adjacent $\mathrm{Au}=\mathrm{O}$ site (purple). b) Rutile $\mathrm{MnO} 2$ without $\mathrm{H}$ transfer (green), with $\mathrm{H}$ transfer (red), with $\mathrm{H}$ transfer to an adjacent $\mathrm{Au}=\mathrm{O}$ acceptor (purple), and with $\mathrm{H}$ transfer to an $\mathrm{Mn}-\mathrm{O}-\mathrm{Au}$ site (blue); blue and purple lines coincide. c) $\mathrm{CoOOH}$ (0112) (green), (0114) (red), and (0001) (purple) surfaces, $\mathrm{Co}_{3} \mathrm{O}_{4}$ (blue), and $\mathrm{Co}_{3} \mathrm{O}_{4}$ with $\mathrm{H}$ transfer to a $\mathrm{Au}=\mathrm{O}$ acceptor (black). Energy levels for an ideal catalyst (dots). ${ }^{93}$

Fortunelli and Goddard III et al. ${ }^{88}$ carried out DFT calculations of the OER on $\mathrm{Pt}$ (111) in the presence of watery electrolyte. In changing the dielectric constant of the electrolyte $(\varepsilon)$, the authors calculated the electrostatic polarization term, assuming that cavitation and dispersion/repulsion contributions to the solvation 
energy do not change. Thus, changing this environmental variable can be used to tune the rate determining steps and the barriers, providing a means for screening and validating new systems to optimize the rate determining steps for the OER reaction pathway. Figure 19 shows that the energy barriers of the individual OER reaction steps on a $\mathrm{Pt}(111)$ surface exhibit a quite diverse behavior as a function of the inverse of the solvent dielectric constant. This implies that the dielectric constant of the electrolyte is an important parameter for improving the OER. With this, the DFT calculations provide a way to extract and validate the mechanistic understanding at the liquid electrolyte - solid electrode interface.

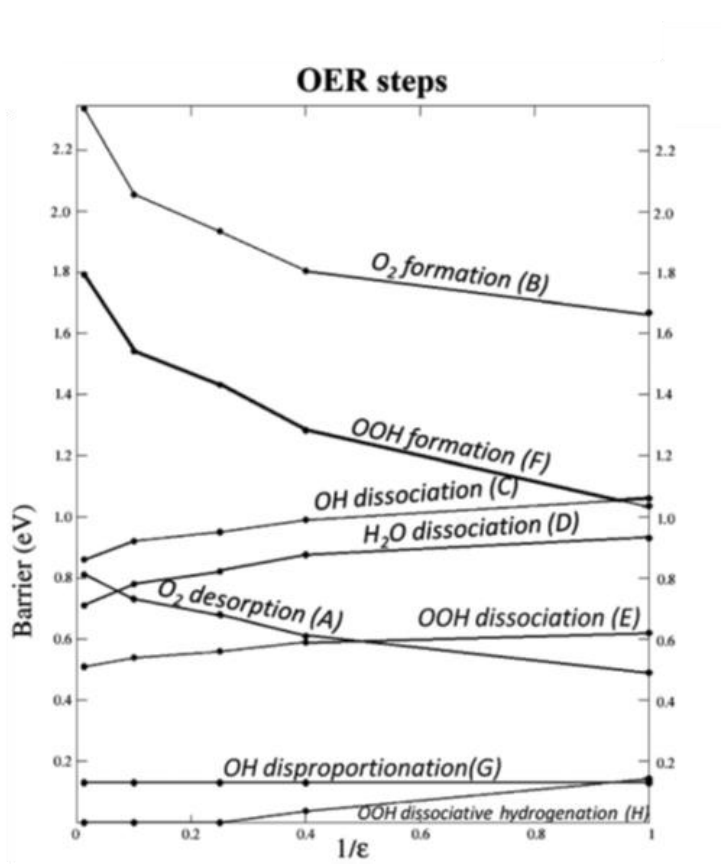

Figure 19. Predicted energy barriers for the individual reaction steps of the OER on $\mathrm{Pt}(111)$ as a function of the inverse of the dielectric constant of the solvent $\varepsilon^{88}$

Bajdich et al. ${ }^{90}$ compared the theoretical overpotentials for three different surfaces of cobalt oxides and found that the (1014) surface is the most active one with the lowest overpotential of $\eta=0.48 \mathrm{~V}(0.8,0.8,0.48 \mathrm{~V}$ for $0001,01 \overline{12}$, and $10 \overline{14}$ surfaces respectively). The high activity of the (1014) surface can be attributed to the observation that the resting state of $\mathrm{Co}$ in the active site is $\mathrm{Co}^{3+}$ during the OER, whereas $\mathrm{Co}$ is in the $\mathrm{Co}^{4+}$ state in the less active surfaces. The overpotential of the (1014) surface can be lowered even further by surface substitution of $\mathrm{Co}$ by $\mathrm{Ni}$. This finding could explain the experimentally observed enhancement in the OER activity of $\mathrm{Ni}_{y} \mathrm{CO}_{1-y} \mathrm{O}_{x}$ thin films with increasing $\mathrm{Ni}$ content. It was found that the natural (0001) facet leads to low OER activity, while higher index surfaces such as $(01 \overline{12})$ or $(01 \overline{14})$, which contain under-coordinated metal sites have more active sites. ${ }^{85,90}$

By means of DFT $+U$ method Nguyen et al. ${ }^{94}$ investigated water oxidation on defective hematite (0001) substrates. The point defects include Fe and $O$ vacancies. They found that iron vacancies do not reduce the OER overpotential and that oxygen vacancies lower the overpotential by $\sim 0.3 \mathrm{~V}$ compared to the ideal case. However, more recently, Hellman et al. ${ }^{76}$ found that the effect of the oxygen vacancy on reducing the overpotential is surface termination dependent. By using first-principle calculations, Hellman et al. studied the oxygen evolution reaction on hydroxyl- and oxygen-terminated hematite. The onset potential was determined to be $1.79 \mathrm{~V}$ and $2.09 \mathrm{~V}$ vs. the reversible hydrogen electrode (RHE) for the pristine hydroxyl and oxygen-terminated hematite, respectively. The presence of oxygen vacancies in the hematite surface resulted in pronounced shifts of the onset potential to $3.09 \mathrm{~V}$ and $1.83 \mathrm{~V}$, respectively. These results indicate that electrochemical water oxidation on hematite occurs more favourably on the oxygenterminated hematite. ${ }^{76}$ Hence, surface termination and non-stoichiometry both significantly allow for tuning of the overpotential for the OER.

The OER overpotential, as a key parameter to rate the electrode materials, has been calculated for many materials. It has been calculated based on electrochemical steps. However, its role is not yet clear when the non-electrochemical steps, such as water addition and oxygen release are considered at working conditions. A recent study by Plaisance and van Santen ${ }^{95}$ considered this by studying the structure sensitivity of the oxygen evolution reaction on several surface terminations, (001), (110) and (311), of $\mathrm{Co}_{3} \mathrm{O}_{4}$ using DFT calculations. The protons and electrons released in these steps are transferred to sites on the surface rather than the bulk electrolyte (protons) and the bulk electrode (electrons). The essential finding is that the relative turnover frequencies for the different surface sites are highly dependent on the overpotential. At low 
overpotentials $(<0.46 \mathrm{~V})$, a dual-Co site on the (001) surface is found to be most active. The $\mathrm{O}_{2}$ release step is rate limiting. At medium overpotentials $(0.46-0.77 \mathrm{~V})$ a similar dual-Co site on the (311) surface is most active. However, a different step, O-O bond formation by water addition, is found to be rate limiting for this surface. A single Co site on the (110) surface is most active at overpotentials that are high enough $(>0.77 \mathrm{~V})$. Thus, the study demonstrated the importance of considering the non-electrochemical steps (water addition and $\mathrm{O}_{2}$ release) rather than only the thermodynamics in modeling the OER.

\section{Application of computational methods in PEC solar fuel conversion}

\subsection{Density functional theory (DFT)}

$\mathrm{DFT}^{19,20}$ has become an essential tool to investigate PEC processes, e.g. calculations of band structures, ${ }^{41-}$ ${ }^{43}$ charge transport, ${ }^{45-47}$ charge transfer, ${ }^{96,97}$ and OER reactions at interfaces. ${ }^{14,57,79-82}$ The applications of DFT in the PEC has been reviewed by Carter et al. ${ }^{14}$ and Oleg et al., ${ }^{12}$ and others. ${ }^{98}$ In particular, Mavros and Voorhis et al. ${ }^{99}$ examined the essential role of DFT in understanding the water-splitting reaction. The authors provided an overview of the current strengths and weaknesses of the state-of-the-art DFT methodologies for condensed-phase molecular simulation involving transition metals and also to guide future experiments and computations toward the understanding and development of novel water-splitting catalysts. The role of DFT in simulations of PEC process is well reviewed in the literature. Thus, in this review we only discuss a few recent examples.

The standard DFT, often fails to describe systems with localized (strongly correlated) $d$ electrons. With standard DFT, errors in the oxidation energies arise due to improper treatment of the d-electrons. ${ }^{19}$ The Hubbard $U$ algorithm is the computationally easiest addition one can use to capture correct reaction energies. ${ }^{44,79} \mathrm{Xu}$, Rossmeisl and Kitchin ${ }^{19}$ presented a DFT+U study on the adsorption of OER intermediates on the (110) surface of rutile. The authors demonstrated a number of universal relationships between the Hubbard $U$ and catalytic processes on transition metal oxides. They evaluated the effect of adding a calculated, linear response $U$ on the predicted adsorption energies, scaling relationships, and overpotential trends with respect to the oxygen evolution reaction for a set of transition metal dioxides. The changes in reaction energy with the application of the calculated $U$ value was found to be on the order of $0.2-0.4 \mathrm{eV}$. It is found that the addition of the Hubbard $U$ greater than zero does not break scaling relationships established without the Hubbard $U$ as shown in Figure 20. The changes in the adsorption energy produced by applying the linear response $U$ for all species are moved toward the weaker binding leg of the volcano plot as guided by the arrows. This means that the universal weakening of adsorption energies is caused by applying the Hubbard U. The addition of the Hubbard $U$ term leads to changes in the relative ordering of activity. The ordering with the addition of the Hubbard $U$ shows better agreement with experiments as discussed in ${ }^{19}$.

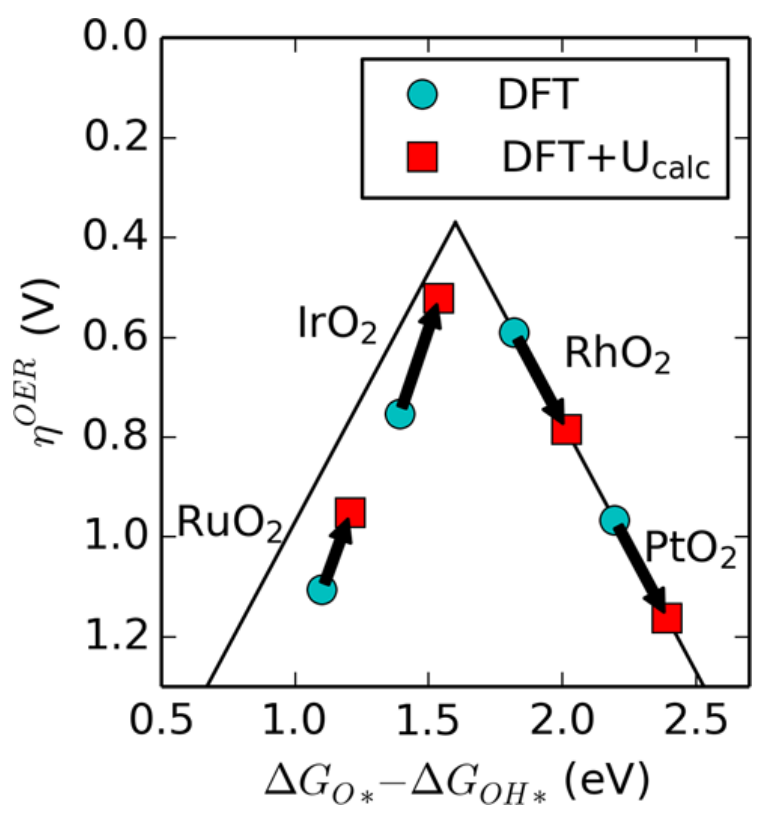

Figure 20. Predicted scaling relationship of $4 d$ and $5 d$ rutile dioxides calculated without (blue circle) and with (red square) the linear response $U$. The arrows point from DFT to DFT+U. ${ }^{19}$ The volcano is fit to the idealized scaling relationships determined in ref ${ }^{100}$

In DFT calculations, the electrochemical charge transfer barriers are usually simulated at constant charge. This leads to potential shifts along the reaction path. ${ }^{84,101,102}$ However, an electrochemical system operates at constant potential, which corresponds to a hypothetical 
model system of infinite size. Previous studies of charge transfer reactions have relied on a computationally costly scheme that extrapolates the barriers calculated on increasingly larger cells. ${ }^{84,101,102}$ It is costly for complicated systems, such as the OER. Recently, Chan and Norskov presented a method that made the DFT calculation of constant potential reaction energetics of electrochemical charge transfer simple. ${ }^{101}$ The method requires a single barrier calculation and the corresponding surface charge at the initial, transition, and final states. The calculation is based on a constant potential correction obtained by charge extrapolation rather than previous cell extrapolation. The constant potential corrections were determined using the energy differences between the work functions of different states (initial state, transition state and final state).

$$
\Delta E_{\phi_{1}-\phi_{2}}=E\left(\phi_{1}\right)-E\left(\phi_{2}\right)=\frac{\left(q_{2}-q_{1}\right)\left(\phi_{2}-\phi_{1}\right)}{?}
$$

where 1 and 2 refer to different states. q1, and q2 are the charges, which can be determined by the Bader analysis.

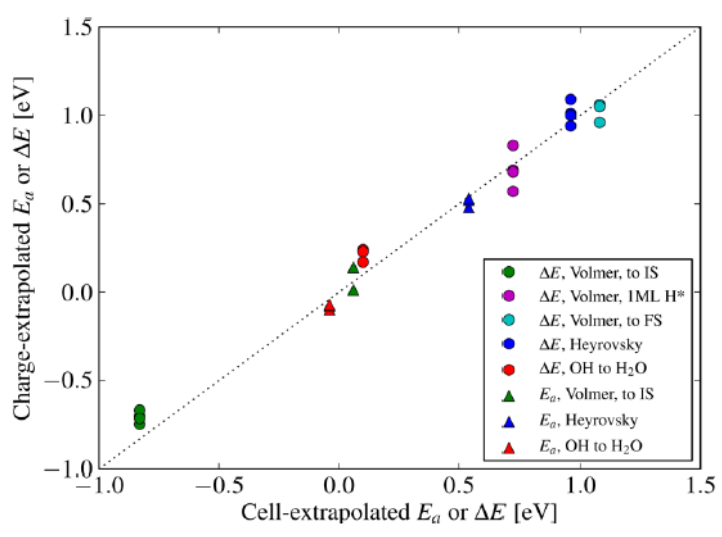

Figure 21. Parity plot of reaction energies and barriers (for three simple elementary proton transfer reactions, Volmer, Heyrovsky, and $\mathrm{OH}$ reduction to $\mathrm{H}_{2} \mathrm{O}$ ) obtained using the cellextrapolation scheme and the charge-extrapolated values. ${ }^{101}$

The comparison of reaction energies and barriers obtained using the cell-extrapolation scheme and the charge-extrapolated values are shown in the parity plot in Figure 21. Regardless of the unit cell size, the simple charge extrapolated scheme gave nearly identical constant potential reaction energetics to that from the cell-extrapolation scheme. However the cellextrapolation scheme is dramatically costlier. This method allows for a tremendous reduction in the computational resources required in DFT calculations of electrochemical barriers. Although it is demonstrated using a simple model system, e.g., the hydrogen evolution reaction, the authors highlighted that this method paves the way for a rigorous DFT-based kinetic analysis of more complex electrochemical reactions. We believe that this will be a promising method to analyse the kinetics of oxygen evolution reactions.

\subsection{Ab-initio molecular dynamics}

The basic concepts and applications of AIMD ${ }^{24-26}$ have been well discussed in ref. ${ }^{24}$ AIMD is particularly suitable for modeling water adsorption, dissociation, deprotonation, and proton transfer at the solid-water interface. Cheng and Sprik et al. ${ }^{103}$ reviewed the application of AIMD in the calculation of redox potentials and acidity constants. The combination of computation of redox potentials and acidity constants allows for calculating the thermochemistry of proton coupled electron transfer (PCET). This is a crucial step in water oxidation, as discussed in the review article. ${ }^{103}$

Spontaneous dissociation of water on defect-free rutile $\mathrm{TiO}_{2}$ (110) was reported by Lindan et al. ${ }^{104}$ to occur in Car-Parrinello molecular dynamics (CPMD) ${ }^{26}$ trajectories at $500 \mathrm{~K}$. The CPMD code is a parallelized plane wave / pseudopotential implementation of Density Functional Theory, particularly designed for ab-initio molecular dynamics. ${ }^{27}$ However, Langel ${ }^{105}$ found that molecular water is stable at $350 \mathrm{~K}$ on the (110) surface, whereas spontaneous dissociation was observed at oxygen vacancies of the defective $\mathrm{TiO}_{2}(100)$ surface. In another CPMD simulation, ${ }^{106}$ Langel and Parrinello showed that isolated water molecules spontaneously dissociate at defective $\mathrm{MgO}(100)$ surfaces, but not on the defect-free surface. Tilocca and Selloni ${ }^{107}$ studied the adsorption of a $\mathrm{H}_{2} \mathrm{O}$ molecule on partially reduced anatase using CPMD simulations. The authors elucidated the dissociation pathway of a water molecule adsorbed close to a low-coordinated defect site on the $\mathrm{TiO}_{2}$ anatase (101) surface as shown in Figure 22. It was found that even though the overall barrier is small, the process is complex, involving a few different intermediate states. 


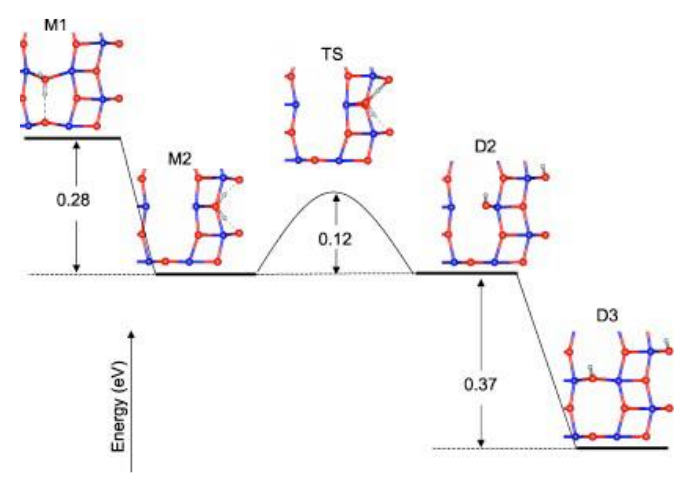

Figure 22. Potential energy diagram for the proposed water dissociation path.

Carravetta et al. studied the adsorption of water on a perfect $\mathrm{TiO}_{2}(110)$ surface by quantum molecular dynamics simulation. ${ }^{108}$ It was found that $\sim 20 \%$ of water molecules dissociate, while the remainder are physisorbed (no dissociation). It agrees rather well with previous estimations, $10 \%-20 \%$, from experimental works. ${ }^{109}$

At the PEC electrode-electrolyte interface, proton transfer occurs accompanied with water oxidation. The dynamics of proton transfer at the oxide-water interface is still not well understood. Tocci and Michaelides ${ }^{110}$ used ab initio molecular dynamics to unravel the connection between interfacial water structure and proton transfer at the water- $\mathrm{ZnO}$ interface. The authors found that upon going from a single layer of adsorbed water to a liquid multilayer, changes in the structure are accompanied by a dramatic increase in the protontransfer rate at the surface. It was found that hydrogen bonding at the interface is responsible for the protontransfer dynamics. The implication of this for modeling PEC processes is that explicit modeling of water molecules is helpful, although most of the literature used the solid-gas interface.

These studies show that ab-initio molecular dynamics simulations undoubtedly represent a powerful tool to explore the dynamics of water on oxide surfaces. Particularly, the solid-water interface is rationally simulated at given temperatures.

\subsection{Multiscale modeling approaches in PEC}

It has been outlined that the multiscale modelling in PEC system is a promising future research direction. ${ }^{12,34,111}$ The operation of photo-electrochemical devices involves many processes occurring simultaneously, competing with or reinforcing each other and spanning a large range of time and length scales. These range from femtosecond to hours, and from angstrom to centimeter. Although a partial elucidation of each of the individual processes has been achieved, we do not yet have a complete, unified picture of the operation of PEC under various conditions. Further progress in the elucidation of the operating principles of photoelectrochemical devices can possibly be achieved with multiscale modeling approaches, which may then bring us closer to the theoretical limit of photocatalytic efficiency. ${ }^{12}$ A multiscale framework is desirable to interrelate phenomena at the relevant time and length scales. ${ }^{34}$ So far, only two level combinations were published; we discuss in the following these multiscale modeling and simulation approaches with two level combinations.

\section{Reactive force field molecular dynamics}

The reactive force field parameterization is usually done via fitting to quantum chemical data, such that the prediction of the chemical reactions are based on a more accurate level of theory (quantum chemical level). It has been applied to many theoretical research fields, such as simulations of mechanical, catalytic, and thermodynamic properties. ${ }^{29,}{ }^{112-115}$ Here, we discuss a few important examples of ReaxFF applied to study water splitting.

The water splitting processes at $\mathrm{TiO}_{2}$ surfaces have been studied by van Duin et al. ${ }^{116}$ The authors optimized the force-field parameters for $\mathrm{TiO}_{2}$-water systems. The training set for optimization of the force field is a collection of results (energies, geometries, charges, etc.) derived from DFT calculations. It consists of equation of state, surface formation, water-binding energy, and $\mathrm{TiO}_{2}$ clusters. The force field determined by DFT data was then applied in MD to study the adsorption and dissociation of water on anatase (101), (100), (112), (001), and rutile (110) at various water coverages. The 
molecular and dissociative adsorption configurations predicted by the ReaxFF for various water coverages agree with previous theoretical studies and experiments. ${ }^{116}$ ReaxFF predicts a complex distribution of water on these surfaces depending on an intricate balance between the spacing of the adsorption sites (under-coordinated $\mathrm{Ti}$ and $\mathrm{O}$ surface atoms), watersurface interactions, and water-water interactions. They also demonstrated a correlation between the extent of water dissociation on different $\mathrm{TiO}_{2}$ surfaces and the strength of hydrogen bonding between adsorbed water molecules and water outside the adsorbed layer, as evidenced by the red shift of the $\mathrm{O}-$ $\mathrm{H}$ vibrational stretching mode of adsorbed water. ${ }^{117}$ Other force fields for oxide-water systems were also successfully developed. Aryanpour et al. ${ }^{118}$ developed two sets of reactive force fields for iron oxides/ oxyhydroxides. Their calculated radial distribution function data is in good agreement with quantum chemical data and that of experimental studies. ${ }^{118,}{ }^{119}$ This confirms the quality of the force field. It is useful for molecular dynamics simulations of water - iron oxides/ oxyhydroxides interactions relevant for semiconductorelectrolyte interface in PEC application.

In another study, Raju and van Duin et al. ${ }^{117}$ found that water in the hydration layer immediately adjacent to the adsorbed water layer enhances water dissociation on the studied anatase surfaces, such that the terminal water dissociation percentage (TWDP) increases with water coverage. In contrast, the TWDP goes through a maximum with water coverage over rutile (110). Stronger hydrogen bonding between the adsorbed water layer and the hydration layer covering this adsorbed layer can account for the increase in TWDP with water coverage over the anatase surfaces.

Using the ReaxFF reactive force field, Russo et al. studied the dynamics associated with the dissociation of water on an aluminum nanocluster. The authors showed that with the assistance of a solvated water molecule, the dissociation of an adsorbed water molecule is possible via a reduction in activation barrier. This reaction occurs in two steps. During the first step, the solvated water removes a hydrogen from the adsorbed water to become an $\mathrm{OH}^{-}($ads $)$and $\mathrm{H}_{3} \mathrm{O}^{+}(\mathrm{g})$.
This newly formed hydronium ion then donates one of its hydrogen atoms to the aluminum surface if a free site is available. ${ }^{120}$ The effect of solvent water molecules in the water splitting process is also demonstrated in another ReaxFF MD study. The hydroxylation structural features of the first adsorption layer and its connection to proton transfer reactivity for the $\mathrm{ZnO} /$ liquid water interface at room temperature have been studied by Raymand and Gorddard et al. ${ }^{121}$ Molecular dynamics simulations employing the ReaxFF forcefield were performed for water on seven $\mathrm{ZnO}$ surfaces with varying step concentrations. Calculations of the free energy barrier for transferring a proton to the surface show that stepped surfaces stabilize the hydroxylated state and decrease the water dissociation barrier. On highly stepped surfaces the barrier is only $2 \mathrm{~kJ} / \mathrm{mol}$ or smaller. The authors compared two models with a monolayer water-ZnO interface and a $\mathrm{ZnO}$-liquid water interface. Figure 23 shows the time dependence of the $\mathrm{OH}$ coverage at the $\mathrm{ZnO} /$ water-interface for the different $\mathrm{ZnO}$ surfaces. Figure 23a (monolayer water-ZnO interface) shows that for the least stepped surface, (1010), the degree of hydroxylation is close to $50 \%$, while for the most stepped surface, (1120), it approaches $80 \%$, with intermediate degrees of hydroxylation for the other surfaces. Compared to monolayer coverage, a higher level of hydroxylation was found for the model of a $\mathrm{ZnO}$-liquid water interface as shown in Figure 23b. For the least stepped surface, (1010), the degree of hydroxylation is $80-85 \%$, while the most stepped surface, (1120), is fully hydroxylated. The other surfaces are hydroxylated to $90 \%$ or more. The authors concluded that this is due to the increased possibility of hydrogen bonding with the water phase outside the first monolayer.
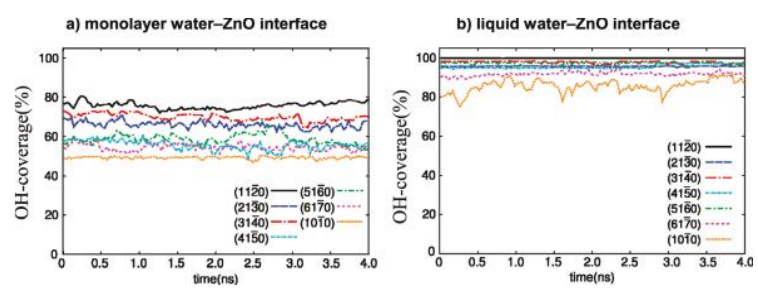

Figure 23. Time dependence of the $\mathrm{OH}$-coverage at the $\mathrm{ZnO}$ /water-interface for the different $\mathrm{ZnO}$ surfaces, (a) the monolayer water-ZnO interface. (b) Liquid water-ZnO interface. ${ }^{121}$ 
Thus, the ReaxFF reactive force field is robust in being able to describe water binding and dissociation on various oxide surfaces in the presence of explicit water molecules (also demonstrating the importance of hydrogen bonding) for a range of water coverages and temperatures. It is therefore suitable for large-scale simulation of oxide-water interfaces in the application of PEC processes. The important role of solvent water molecules in reducing the activation energy of water splitting has been demonstrated in a few ReaxFF MD simulations as discussed above. This effect is usually missed in DFT calculations.

\section{Kinetic Monte Carlo (kMC)}

$\mathrm{KMC}^{30-32}$ has been used in many fields that are relevant to the PEC processes, ${ }^{30,34,45,122,123}$ such as simulation of charge transport in the semiconductor electrode, ${ }^{45,124}$ electrochemical reactions at the electrode surface, ${ }^{122}$, 125,126 and analysis of electrochemical impedance spectra. 31,127

KMC was employed in charge transport calculations by the group of Rosso et al. ${ }^{45,124}$ In a first step, the rate of electron transfer in bulk hematite was obtained by ab initio electronic structure calculations (DFT). The DFT results were then used for modeling the charge transport in hematite by $\mathrm{kMC}$. By analysing the $\mathrm{kMC}$ simulated results, the authors found that defects significantly affect electron polaron diffusion at low polaron to defect ratios due to trapping on nanosecond to microsecond time scales. ${ }^{45}$ Figure 24 compares the diffusion coefficient curve obtained with the defective cells with that from the pure simulations. Due to the attractive electrostatic interactions between electron polarons and the positively charged defects, the electron polarons reside around the defects for some period of time, thus slowing down the overall polaron diffusion. When the polaron to defect ratio is low, a significant proportion of the electron polarons is trapped at defect sites and the diffusion coefficient is greatly reduced as shown in Figure 24. As the polaron to defect ratio increases, a greater proportion of the polarons is not bound and the diffusion coefficient increases. At the same time, as the polaron concentration increases, the repulsive electron polaron-electron polaron interactions reduce the diffusion coefficient. The diffusion coefficient curve therefore reaches a plateau before decreasing again and becoming similar to the case where no defects are present.

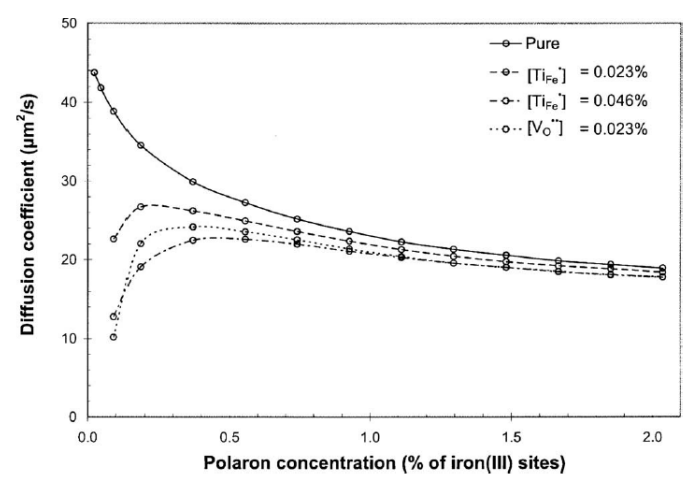

Figure 24. Electron polaron diffusion coefficient as a function of polaron concentration for several positively charged defects. ${ }^{45}$

Viswanathan and Norskov et al. ${ }^{128}$ presented a multiscale model for simulation of linear sweep voltammetry of electrochemical oxidation of water on $\mathrm{Pt}(111)$ and $\mathrm{Pt}_{3} \mathrm{Ni}(111)$. DFT was used to parameterize the reaction kinetics and $\mathrm{kMC}$ was used to capture the kinetic steps of electrochemical reactions. The calculated voltammogram is in good agreement with the experimental result. ${ }^{128,129}$ The theoretical calculations predicted that $\mathrm{OH}$ adsorbs between $0.65 \mathrm{~V}_{\mathrm{RHE}}$ and 0.85 $\mathrm{V}_{\mathrm{RHE}}$. At $0.9 \mathrm{~V}_{\mathrm{RHE}}, \mathrm{OH}$ starts to get oxidized to $\mathrm{O}$.

\section{State space modelling (SSM)}

SSM is a well-known method in control theory to simulate complex, interdepending systems. ${ }^{33}$ The statespace representation is a mathematical model of a physical system as a set of input, output and state variables related by first-order differential equations. The state variables can be reconstructed from the measured input-output data, but are not themselves measured during the experiment. The general statespace representation is given as follows

$\dot{\boldsymbol{x}}(t, \boldsymbol{p})=\frac{d \boldsymbol{x}(t, \boldsymbol{p})}{d t}=\mathrm{f}[\boldsymbol{x}(t, \boldsymbol{p}), \boldsymbol{u}(t), t, \boldsymbol{p}]$ 
where $\mathbf{x}(t, \mathbf{p})$ represents the vector of the state-variables depending on the time $t$, and the vector of unknown parameters, $\mathbf{p}$. The vector $\mathbf{u}(\mathrm{t})$ signifies the input variables that can be varied in the simulations. In addition to the differential equations (Eq. (1)), the statespace description contains the observation function $\mathbf{y}(\mathrm{t}, \mathbf{p})$, which denotes the observed quantities and is referred to as the model output.

$\boldsymbol{y}(t, \boldsymbol{p})=\boldsymbol{g}[\boldsymbol{x}(t, \boldsymbol{p}), \boldsymbol{p}]$

State-space modeling was used in a few examples for electrochemical systems to calculate impedance spectra, current - voltage plots and Tafel plots in fuel cells and batteries. ${ }^{130-135}$

In ref ${ }^{132}$, we have shown for the anodic SOFC (solid oxide fuel cell) system $\mathrm{Ni}, \mathrm{H}_{2}-\mathrm{H}_{2} \mathrm{O}$ |YSZ (yttria-stabilized zirconia) that an electrochemical model can be identified with such a state space representation. When mass and charge balances are formulated from an electrochemical model, the surface concentrations of the different adsorbed species are the vector of state variables, $\mathbf{x}(\mathrm{t}, \mathbf{p})$. The concentration of the adsorbed species is changing as a function of time, $t$, and as a function of diverse other parameters, $\mathbf{p}$, such as surface coverage and reaction rate constants. The applied potential is the input variable, $\mathbf{u}(\mathrm{t})$, that can be changed in the experiments, and the Faraday current is the model output, $\mathbf{y}(\mathrm{t}, \mathbf{p})$.

By implementing the electrochemical model as described in ref $^{132}$ in MATLAB and SIMULINK, the impedance at the $\mathrm{Ni}, \mathrm{H}_{2}-\mathrm{H}_{2} \mathrm{O}$ YSSZ interface was simulated and compared to experimental measurements under the same conditions. Required input values were at first instance derived with the help of the literature. Fitting the simulated data to experimental measurements under standard conditions allowed optimizing the input values. Figure 25 shows experimental and simulated impedance spectra. The simulated impedance yields values in the same order of magnitude as the experimental ones and the spectrum exhibits one arc, such as in the experiment. The deviation between experiment and simulation is mainly attributed to the availability of kinetic input data. Such data can be determined by atomic modeling, such as
DFT. Also, diffusion effects can be implemented in the SSM based on kinetic Monte Carlo simulations. In this sense, the SSM approach is a multi-scale modeling and simulation approach. This potential was, however, never fully taped in the field of fuel cell systems where this approach was used for the first time for an electrochemical system. It has never been applied for PEC systems so far. In section 5, we propose to transfer SSM to PEC systems and to use it as one level of a multi-scale approach.

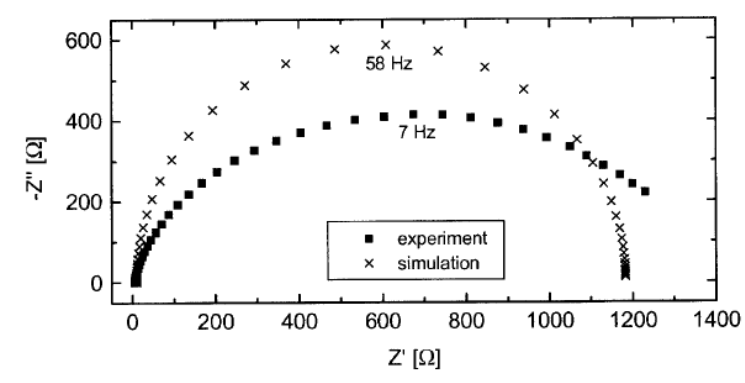

Figure 25. Experimental and simulated impedance spectrum of the $\mathrm{Ni}, \mathrm{H}_{2}-\mathrm{H}_{2} \mathrm{O} \mid \mathrm{YSZ}$ interface. ${ }^{132}$

\section{Computational challenges}

1 Many DFT simulations of free energies were carried out in the past to study the oxygen evolution reaction. ${ }^{14,44,57,59,76,79,136}$ While pragmatic and useful for initial screening of electrode materials, a severe limitation is that the reaction barriers are not calculated and hence no information about the kinetics is gained from these simulations. ${ }^{99}$ Although calculations of activation energies were published in a few papers, ${ }^{61,80,101}$ it is still a challenge to search for the transition state, especially when the water molecules in solution are considered explicitly. Furthermore, the non-electrochemical steps, such as water addition (the addition of one water molecule to the electrode surface can be a physical adsorption or chemically lead to displacement of the surface species coupled with intramolecular proton transfer, resulting in terminal species such as $\mathrm{OOH}$ or $\mathrm{OH}$ groups) and $\mathrm{O}_{2}$ desorption, are usually not considered 
in these calculations. Voorhis et al. ${ }^{99}$ found a very low barrier for $\mathrm{O}-\mathrm{O}$ bonding, suggesting that the identity of the rate-determining step may be different from generally expected (for example, water addition or intramolecular proton transfer). Recently, experimental evidence of temperature-programmed desorption demonstrated that the interaction between $\mathrm{O}_{2}$ and water may pose a bottleneck in the evolution of $\mathrm{O}_{2}$ from the catalyst surface during water oxidation. ${ }^{137}$ The challenge is searching for the rate determining step in the simultaneous consideration of electrochemical and non-electrochemical steps under PEC working conditions.

2 Most of the DFT calculations have been done using a solid-gas model.44, 57, 59, 76, 94, 136, 138 However, in a PEC system, the working condition is a solid-liquid interface. To account for this, models with monolayers of water have been used. ${ }^{80,}$ 139-142 Carter et al. compared monolayer models with vacuum models and found very little effect of monolayer water on free energy calculations. ${ }^{139}$ DFT calculations with explicit water (more than one overlayer) models have also been performed. Such models with explicit inclusion of water molecules are more realistic to simulate the electrode-electrolyte interface, however, they are less common and computationally more expensive. ${ }^{62,69,143}$ thus, simulated interfaces differ often significantly from the real system. Therefore, it is of great importance to bridge the gap between the model systems used in computer simulations and true systems, exposed to realistic operating conditions. Resolving this issue is not just a matter of adding more atoms to the simulations in order to make the molecular model more realistic; it is actually a fundamental challenge as discussed by Rossmeisl et al. ${ }^{81}$ They showed that none of the existing methods deal with all the thermodynamic constraints that the electrochemical environment imposes on the structure of the interface. The challenge is to realistically simulate the electrochemical interface including potential, $\mathrm{pH}$ and counterions. $^{81}$

3 There are many mechanisms proposed for the OER as discussed in section 2. However, common agreement has not been achieved yet. Searching for new mechanisms that can well reproduce experimental measurements is demanding. The challenge is how to confirm the proposed mechanism by experimental evidence. Although some mechanisms have been demonstrated to some extend to be in agreement with experimental results, there are still many uncertainties, due to limitations of both experimental techniques (in solution) and modeling methods (for the solid-liquid interface as mentioned above). New approaches are required to bring experiment and theory together.

4 Many reactions in the PEC process take place at the solid-liquid interfaces. Rate limiting reactions have been identified for many materials. While the charge transport within the semiconductor can also limit the surface reactions, it is important to find out which process is rate limiting (within the semiconductor or at the interface) in the whole PEC process. The challenge is how to couple semiconductor physics with surface chemistry which considers simultaneously charge transport, charge transfer and surface reactions.

5 Two-level multiscale methods have been recently used as discussed in chapter 3 . However, building up a many-level multiscale modelling approach for such a complex system consisting of multiple reactions and species as well as many interfaces is challenging. This is due to the complexity and multidisciplinary of the PEC process and the fact that it spans a few time and length scales. 
6 The photoelectrochemical reactions were studied mostly under electrochemical conditions. The light-matter interaction is mainly done for optical absorptions of the photoelectrode. The light induced water splitting reactions of photocatalytic process, in which the photoexcitation is treated explicitly as the driving force of the water splitting reaction, have only been employed in small model systems. ${ }^{38,40}$

\section{Conclusions and Outlook}

This paper reviews recent modeling and simulation efforts on the photo-electrochemical interface. Computational methods with respect to photoelectrochemical modeling and simulations are summarized and discussed specifically regarding PEC systems. Finally, the computational challenges with respect to these systems are discussed.

It can be concluded that the efforts on computational studies of the PEC interface are increasing lately. Most efforts are related to single aspects of the PEC interface such as the band gap and the tailoring of the band gap, the charge transport, or the electrochemical reactions. The simulations are usually not directly compared to experimental PEC measurements which is related to the complexity of the interface and the complex experimental conditions, such as light and liquid-solid interface, which are difficult to consider in simulations. No consensus was found so far about the oxygen evolution reaction mechanism at these PEC interfaces. Hence, a multiscale modeling and simulation approach is required in order to connect the different efforts and to relate the modeling and simulation results directly to the experiments. Usually, PEC data, in particular impedance data, are fitted to so-called equivalent circuits. After fitting of the measurement data to an equivalent circuit model, resistances, capacitances, and inductances are obtained which represent the impedance data well. However, that phenomenological data is not directly related to electrochemical quantities. On the other side, computational results from modeling and simulations, such as free energies, do not directly represent the experimental data. We therefore suggest an approach which can close this gap.

The approach that we are suggesting spans four levels of theories which are well coupled. The inputs and outputs of each level are presented in Figure 26. In the first level, density functional theory (DFT) will be used to simulate, at atomistic level, the reactions at the electrode-electrolyte interface. This includes the calculation of the Gibbs free energies of each reaction step from which the overpotential for the reaction can be derived. Additionally, transition states, activation energies, and rate constants will be calculated. In the second level, reactive force field molecular dynamics (ReaxFF) will be used to simulate, at the molecular level, the restructuring of the semiconductor surface and / or the co-catalyst. DFT calculated data will be used to train a suitable reactive force field for the given system. The restructuring induced changes in catalytic activities can be further determined by DFT calculations. In the third level, kinetic Monte Carlo ( $\mathrm{kMC}$ ) is used to simulate multiple reactions at the interfaces (including adsorption, diffusion, reaction, and desorption). The rate constants for these processes are determined from DFT and molecular dynamics simulations. The simulations will allow for determining the rate limiting reaction step, charge transport, and coverage dependence.

In the fourth level, continuum level, state space modeling (SSM) allows for simulating PEC data that can be directly compared to the experiments. The inputs of SSM are calculated by the other levels of methods as mentioned above. Using an optimization procedure with experimental data, the input data can be optimized and kinetic parameters can be determined. Then the validation and verification will be done based on matching with experimental data measured under diverse operating conditions. How this works, is described with the following example: In the experimental impedance data, a large arc appears, i.e. an unknown process has a high impedance and is therefore limiting the performance. The same arc is observed in the simulated impedance data and can be traced back to a specific process, e.g. the desorption of oxygen, based on the assumed electrochemical model. In order to verify the assignment of the impedance arc 
to this specific electrochemical process, parameters both in the experiment and in the simulation are modified, e.g. by surface treatment of the electrode in the experiment to improve desorption and modification of the desorption rate in the simulation, respectively. If similar trends are observed, the assignment of the impedance arc is confirmed and desorption is identified as a performance limiting process here. Measures can then be taken to design an electrode with higher performance. For the case of not matching with experimental data, the electrochemical model, assumptions in the simulations, and / or kinetic parameters have to be revised and modified. The DFT calculated data will be further optimized to fit the experimental measurements. In the worst case, a new electrochemical model has to be set up. Several cycles of optimization and re-evaluation might be necessary to achieve agreement between experiment and simulation.

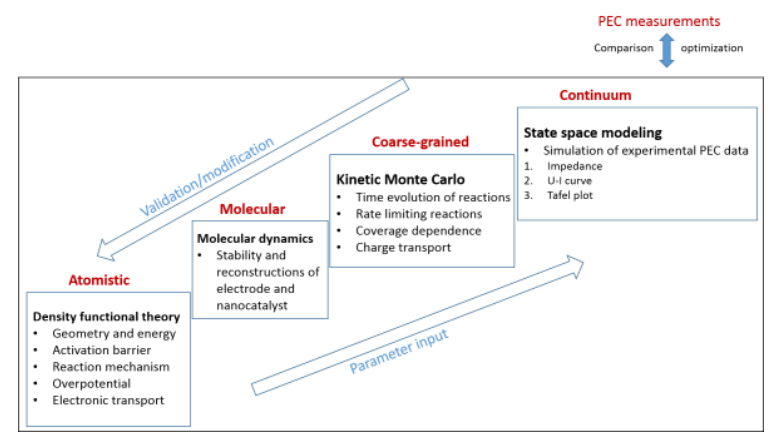

Figure 26. Suggested multiscale modeling and simulation approach for PEC interfaces including four levels of theory. The system size and time scale that can be simulated increase from the left to the right.

With this approach many details of the reaction mechanism taking place at the PEC interface can be extracted, and the rate limitations in the PEC process can be identified. This is a versatile multiscale modelling approach particularly suitable for complex systems. Except for PEC system, the approach can be widely applied to other systems, such as fuel cells, electrolyser, batteries, sensors, etc.

\section{Acknowledgement}

Zhang acknowledges the financial support from DIFFER for carrying out this study. The authors thank Dr. Peter Klaver, Dr. Paola Diomede, Dr. Egbert
Westerhof (DIFFER) and Prof. Rutger van de Santen (TU/e) for carefully proof reading the manuscript.

\section{References}

1. M. Gratzel, Nature, 2001, 414, 338-344.

2. H. M. Chen, C. K. Chen, R.-S. Liu, L. Zhang, J. Zhang and D. P. Wilkinson, Chemical Society Reviews, 2012, 41, 5654-5671.

3. T. M. Gür, S. F. Bent and F. B. Prinz, The Journal of Physical Chemistry C, 2014, 118, 21301-21315.

4. Z. Li, W. Luo, M. Zhang, J. Feng and Z. Zou, Energy \& Environmental Science, 2013, 6, 347-370.

5. K. Maeda and K. Domen, The Journal of Physical Chemistry Letters, 2010, 1, 2655-2661.

6. D. G. Nocera, Accounts of Chemical Research, 2012, 45, 767-776.

7. L. R. Sheppard and J. Nowotny, Advances in Applied Ceramics, 2007, 107, 9.

8. Y. Tachibana, L. Vayssieres and J. R. Durrant, Nat Photon, 2012, 6, 511-518.

9. A. Fujishima and K. Honda, Nature, 1972, 238, 3738.

10. M. M. May, H.-J. Lewerenz, D. Lackner, F. Dimroth and T. Hannappel, Nat Commun, 2015, 6

11. J. R. Bolton, S. J. Strickler and J. S. Connolly, Nature, 1985, 316, 495-500.

12. A. V. Akimov, A. J. Neukirch and O. V. Prezhdo, Chemical Reviews, 2013, 113, 4496-4565.

13. R. van de Krol, Y. Liang and J. Schoonman, Journal of Materials Chemistry, 2008, 18, 2311-2320.

14. P. Liao and E. A. Carter, Chemical Society Reviews, 2013, 42, 2401-2422.

15. A. Valdes, J. Brillet, M. Gratzel, H. Gudmundsdottir, H. A. Hansen, H. Jonsson, P. Klupfel, G.-J. Kroes, F. Le Formal, I. C. Man, R. S. Martins, J. K. Norskov, J. Rossmeisl, K. Sivula, A. Vojvodic and M. Zach, Physical Chemistry Chemical Physics, 2012, 14, 4970.

16. Y. Ping, D. Rocca and G. Galli, Chemical Society Reviews, 2013, 42, 2437-2469.

17. H. Dau, C. Limberg, T. Reier, M. Risch, S. Roggan and P. Strasser, ChemCatChem, 2010, 2, 724-761.

18. J. K. Nørskov and T. Bligaard, Angewandte Chemie International Edition, 2013, 52, 776-777.

19. Z. Xu, J. Rossmeisl and J. R. Kitchin, The Journal of Physical Chemistry C, 2015, 119, 4827-4833.

20. J. K. Norskov, T. Bligaard, J. Rossmeisl and C. H. Christensen, Nat Chem, 2009, 1, 37-46.

21. R. G. Parr and W. Yang, Density-Functional Theory of Atoms and Molecules, Oxford University Press, Oxford, 1989.

22. G. Onida, L. Reining and A. Rubio, Reviews of Modern Physics, 2002, 74, 601-659.

23. F. De Angelis, C. Di Valentin, S. Fantacci, A. Vittadini and A. Selloni, Chemical Reviews, 2014, 114, 97089753.

24. D. Marx and J. Hutter, Ab initio Molecular Dynamics - Basic Theory and Advanced Methods, Cambridge University Press, Cambridge, 2009.

25. E. T. Mark, Journal of Physics: Condensed Matter, 2002, 14, R1297.

26. R. Car and M. Parrinello, Physical Review Letters, 1985, 55, 2471-2474.

27. http://www.cpmd.org/.

28. D. C. Rapaport, The Art of Molecular Dynamics Simulations, Cambridge University Press, Cambridge, U.K., 1995.

29. A. C. T. van Duin, S. Dasgupta, F. Lorant and W. A. Goddard, The Journal of Physical Chemistry A, 2001, 105, 9396-9409.

30. A. P. J. Jansen, An Introduction to Kinetic Monte Carlo Simulations of Surface Reactions, 2012, Springer. 
31. K. B. L. edited by Abby L. Parrill, Reviews in Computational Chemistry, 2015, 28.

32. H. M. Cuppen, L. J. Karssemeijer and T. Lamberts, Chemical Reviews, 2013, 113, 8840-8871.

33. J. D. P. G. F. Franklin, and A. E. Naeini, Feedback Control of Dynamic Systems, 1994.

34. D. F. Jonathan E. Mueller, and Timo Jacob, Electrocatalysis: Theoretical Foundations and Model Experiments, First Edition. Edited by Richard C. Alkire, Ludwig A. Kibler, Dieter M. Kolb, and Jacek Lipkowski, Multiscale Modeling of Electrochemical Systems 2013, Wiley-VCH Verlag GmbH \& Co. KGaA.

35. A. Dreuw and M. Head-Gordon, Journal of the American Chemical Society, 2004, 126, 4007-4016.

36. M. A. L. Marques and S. Botti, The Journal of Chemical Physics, 2005, 123, 014310.

37. D. Rocca, Y. Ping, R. Gebauer and G. Galli, Physical Review B, 2012, 85, 045116.

38. A. Kazaryan, R. van Santen and E. J. Baerends, Physical Chemistry Chemical Physics, 2015, 17, 20308-20321.

39. A. R. a. E. J. B. T. Ziegler, Chem. Phys., 1976, 16, 209-217.

40. E. Berardo, H.-S. Hu, S. A. Shevlin, S. M. Woodley, K. Kowalski and M. A. Zwijnenburg, Journal of Chemical Theory and Computation, 2014, 10, 11891199.

41. F. Wang, C. Di Valentin and G. Pacchioni, The Journal of Physical Chemistry C, 2012, 116, 89018909.

42. Y. Gai, J. Li, S.-S. Li, J.-B. Xia and S.-H. Wei, Physical Review Letters, 2009, 102, 036402.

43. D. K. Kanan and E. A. Carter, The Journal of Physical Chemistry C, 2012, 116, 9876-9887.

44. M. C. Toroker and E. A. Carter, Journal of Materials Chemistry A, 2013, 1, 2474-2484

45. S. Kerisit and K. M. Rosso, The Journal of Chemical Physics, 2007, 127, 124706.

46. V. Viswanathan, K. L. Pickrahn, A. C. Luntz, S. F. Bent and J. K. Nørskov, Nano Letters, 2014, 14, 5853-5857.

47. P. Liao, M. C. Toroker and E. A. Carter, Nano Letters, 2011, 11, 1775-1781.

48. N. Alidoust and E. A. Carter, Physical Chemistry Chemical Physics, 2015, 17, 18098-18110.

49. N. Adelstein, J. B. Neaton, M. Asta and L. C. De Jonghe, Physical Review B, 2014, 89, 245115

50. M. K. Aydinol, A. F. Kohan, G. Ceder, K. Cho and J. Joannopoulos, Physical Review B, 1997, 56, 13541365.

51. M. Kneiß, M. Jenderka, K. Brachwitz, M. Lorenz and M. Grundmann, Applied Physics Letters, 2014, 105 062103.

52. N. A. Deskins and M. Dupuis, Physical Review B, 2007, 75, 195212.

53. I. G. Austin and N. F. Mott, Advances in Physics, 2010, 50, 757-812

54. R. A. Marcus, Reviews of Modern Physics, 1993, 65 599-610.

55. M. C. Toroker and E. A. Carter, The Journal of Physical Chemistry C, 2012, 116, 17403-17413.

56. F. Le Formal, E. Pastor, S. D. Tilley, C. A. Mesa, S. R. Pendlebury, M. Grätzel and J. R. Durrant, Journal of the American Chemical Society, 2015, 137, 66296637.

57. Á. Valdés, Z. W. Qu, G. J. Kroes, J. Rossmeisl and J. K. Nørskov, The Journal of Physical Chemistry C 2008, 112, 9872-9879.

58. A. L. J. Rossmeisl, J.K. Nørskov, Chem. Phys., 2005, 319, 178-184.

59. Z. W. Q. J. Rossmeisl, H. Zhu, G. J. Kroes and J. K Nørskov, J. Electroanal. Chem., 2007, 607, 83-98.

60. Y.-F. Li and Z.-P. Liu, Journal of the American Chemical Society, 2011, 133, 15743-15752.
61. G.-F. Wei, Y.-H. Fang and Z.-P. Liu, The Journal of Physical Chemistry C, 2012, 116, 12696-12705.

62. J. Chen, Y.-F. Li, P. Sit and A. Selloni, Journal of the American Chemical Society, 2013, 135, 1877418777.

63. R. Nakamura and Y. Nakato, Journal of the American Chemical Society, 2004, 126, 1290-1298.

64. Y. Ma, X. Wang, Y. Jia, X. Chen, H. Han and C. Li, Chemical Reviews, 2014, 114, 9987-10043.

65. R. H. Wilson, Journal of The Electrochemical Society, 1980, 127, 228-234.

66. P. Salvador and C. Gutierrez, The Journal of Physical Chemistry, 1984, 88, 3696-3698.

67. R. Nakamura, T. Tanaka and Y. Nakato, The Journal of Physical Chemistry B, 2004, 108, 10617-10620.

68. T. B. J.K. Nørskov, A. Logadottir, S. Bahn, L.B. Hansen, M. Bollinger, H. Bengaard, B. Hammer, Z. Sljivancanin, M. Mavrikakis, Y. Xu, S. Dahl, C.J.H. Jacobsen, J. Catal. , 2002, 209 275-278.

69. Y.-H. Fang and Z.-P. Liu, Journal of the American Chemical Society, 2010, 132, 18214-18222.

70. J. O. Bockris and T. Otagawa, The Journal of Physical Chemistry, 1983, 87, 2960-2971.

71. A. I. Krasil'shchikov, Zh. Fiz. Khim., 1963, 37, 531.

72. C. I. W. O'Grady, J. Huang, and E. Yeager M. W. Brieter, Ed.,, Electrocatalysis.

73. G. H. J. B. A.G.C. Kobussen, J. Electroanal. Chem., 1981, 126, 221

74. M. P. B. Michael E. G Lyons, Int. J. Electrochem. Sci., 2008, 31386

75. M. P. B. Michael E. G Lyons, Int. J. Electrochem. Sci., 2008, 3, 1425.

76. B. I. Anders Hellman, Björn Wickman, Henrik Grönbeck, Jonas Baltrusaitis, Surface Science, 2015, 640, 45-49.

77. J. Yang, H. An, X. Zhou and C. Li, The Journal of Physical Chemistry C, 2015, 119, 18487-18494.

78. P. E. M. Siegbahn, Biochimica et Biophysica Acta, 2013, 1827, 1003-1019.

79. Á. Valdés and G.-J. Kroes, The Journal of Chemical Physics, 2009, 130, 114701.

80. A. Roudgar, M. Eikerling and R. van Santen, Physical Chemistry Chemical Physics, 2010, 12, 614-620.

81. M. E. B. Malte Nielsen, Martin H Hansen, Jan Rossmeisl, Surface Science, 2015, 631, 2-7.

82. S. Haghighat and J. M. Dawlaty, The Journal of Physical Chemistry C, 2015, 119, 6619-6625.

83. J. K. Nørskov, J. Rossmeisl, A. Logadottir, L. Lindqvist, J. R. Kitchin, T. Bligaard and H. Jónsson, The Journal of Physical Chemistry B, 2004, 108, 17886-17892.

84. J. Rossmeisl, E. Skúlason, M. E. Björketun, V. Tripkovic and J. K. Nørskov, Chem. Phys. Lett., 2008, 466, 68-71.

85. D. Friebel, M. W. Louie, M. Bajdich, K. E. Sanwald, Y. Cai, A. M. Wise, M.-J. Cheng, D. Sokaras, T.-C. Weng, R. Alonso-Mori, R. C. Davis, J. R. Bargar, J. K. Nørskov, A. Nilsson and A. T. Bell, Journal of the American Chemical Society, 2015, 137, 1305-1313.

86. J. Cheng, X. Liu, J. A. Kattirtzi, J. VandeVondele and M. Sprik, Angewandte Chemie, 2014, 126, 1224212246.

87. B. M. Szyja and R. A. van Santen, Physical Chemistry Chemical Physics, 2015, 17, 1248612491.

88. A. Fortunelli, W. A. Goddard lii, L. Sementa and G. Barcaro, Nanoscale, 2015, 7, 4514-4521.

89. Y.-F. Li, Z.-P. Liu, L. Liu and W. Gao, Journal of the American Chemical Society, 2010, 132, 1300813015.

90. M. Bajdich, M. García-Mota, A. Vojvodic, J. K. Nørskov and A. T. Bell, Journal of the American Chemical Society, 2013, 135, 13521-13530. 
91. M. García-Mota, M. Bajdich, V. Viswanathan, A. Vojvodic, A. T. Bell and J. K. Nørskov, The Journal of Physical Chemistry C, 2012, 116, 21077-21082.

92. R. S. Khnayzer, M. W. Mara, J. Huang, M. L. Shelby, L. X. Chen and F. N. Castellano, ACS Catalysis, 2012, 2, 2150-2160.

93. R. Frydendal, M. Busch, N. B. Halck, E. A. Paoli, P. Krtil, I. Chorkendorff and J. Rossmeisl, ChemCatChem, 2015, 7, 149-154.

94. M.-T. Nguyen, S. Piccinin, N. Seriani and R. Gebauer, ACS Catalysis, 2015, 5, 715-721.

95. C. P. Plaisance and R. A. van Santen, Journal of the American Chemical Society, 2015, 137, 1466014672.

96. W. R. Duncan, C. F. Craig and O. V. Prezhdo, Journal of the American Chemical Society, 2007, 129, 8528-8543.

97. M. T. M. Koper, Chemical Science, 2013, 4, 27102723.

98. Y. Jiao, Y. Zheng, M. Jaroniec and S. Z. Qiao, Chemical Society Reviews, 2015, 44, 2060-2086.

99. M. G. Mavros, T. Tsuchimochi, T. Kowalczyk, A. Mclsaac, L.-P. Wang and T. V. Voorhis, Inorganic Chemistry, 2014, 53, 6386-6397.

100. I. C. Man, H.-Y. Su, F. Calle-Vallejo, H. A. Hansen, J. I. Martínez, N. G. Inoglu, J. Kitchin, T. F. Jaramillo, J. K. Nørskov and J. Rossmeisl, ChemCatChem, 2011, 3, 1159-1165.

101. K. Chan and J. K. Nørskov, The Journal of Physical Chemistry Letters, 2015, 6, 2663-2668.

102. E. Skúlason, V. Tripkovic, M. E. Björketun, S Gudmundsdóttir, G. Karlberg, J. Rossmeisl, T. Bligaard, H. Jónsson and J. K. Nørskov, The Journal of Physical Chemistry C, 2010, 114, 18182-18197.

103. J. Cheng, X. Liu, J. VandeVondele, M. Sulpizi and M. Sprik, Accounts of Chemical Research, 2014, 47, 3522-3529.

104. N. M. H. P. J. D. Lindan, J. M. Holender, and M. J. Gillan, Chem. Phys. Lett., 1996, 261, 246.

105. W. Langel, Surf. Sci., 2002, 496, 141.

106. W. Langel and M. Parrinello, The Journal of Chemical Physics, 1995, 103, 3240-3252.

107. A. Tilocca and A. Selloni, The Journal of Chemical Physics, 2003, 119, 7445-7450.

108. W. Zhang, J. Yang, Y. Luo, S. Monti and V. Carravetta, The Journal of chemical physics, 2008, 129, 064703

109. M. D. Brinkley, T. Engel, P. Farrall, G. Gantner, A Schafer, and A. Szuchmacher, Surf. Sci. , 1998, 395, 292.

110. G. Tocci and A. Michaelides, The Journal of Physical Chemistry Letters, 2014, 5, 474-480.

111. M. Shishkin and T. Ziegler, Physical Chemistry Chemical Physics, 2014, 16, 1798-1808.

112. K. Joshi, A. C. T. van Duin and T. Jacob, Journal of Materials Chemistry, 2010, 20, 10431-10437.

113. E. C. Neyts, Y. Shibuta, A. C. T. van Duin and A. Bogaerts, ACS Nano, 2010, 4, 6665-6672.

114. E. lype, M. Hütter, A. P. J. Jansen, S. V. Nedea and C. C. M. Rindt, Journal of Computational Chemistry, 2013, 34, 1143-1154.

115. X.-Q. Zhang, R. A. van Santen and E. J. M. Hensen, ACS Catalysis, 2015, 5, 596-601.

116. S.-Y. Kim, N. Kumar, P. Persson, J. Sofo, A. C. T. van Duin and J. D. Kubicki, Langmuir, 2013, 29, 7838-7846.
117. M. Raju, S.-Y. Kim, A. C. T. van Duin and K. A. Fichthorn, The Journal of Physical Chemistry C, 2013, 117, 10558-10572.

118. M. Aryanpour, A. C. T. van Duin and J. D. Kubicki, The Journal of Physical Chemistry A, 2010, 114, 6298-6307.

119. F. Paesani and G. A. Voth, The Journal of Physical Chemistry B, 2009, 113, 5702-5719.

120. Michael F. Russo Jr, Rong Li, Matthew Mench, Adri C.T. van Duin, International Journal of Hydrogen Energy, 2011, 36, 5828-5835.

121. D. Raymand, A. C. T. van Duin, W. A. Goddard, K. Hermansson and D. Spångberg. The Journal of Physical Chemistry C, 2011, 115, 8573-8579.

122. M. T. M. Koper, A. P. J. Jansen, R. A. van Santen, J. J. Lukkien and P. A. J. Hilbers, The Journal of Chemical Physics, 1998, 109, 6051-6062.

123. C. H. T. Kah Chun Lau, Brett I. Dunlap, Solid State Ionics, 2008, 179, 1912-1920.

124. J. Yu, M. L. Sushko, S. Kerisit, K. M. Rosso and J. Liu, The Journal of Physical Chemistry Letters, 2012, 3, 2076-2081.

125. J. J. L. Marc T.M Koper, Journal of Electroanalytical Chemistry, 2000, 485, 161-165.

126. A. P. v. B. C.G.M. Hermse, M.T.M. Koper,J.J. Lukkien, R.A. van Santen, A.P.J. Jansen, Surface Science, 2004, 572, 247-260.

127. S.-I. P. Sung-Woo Kim, Journal of Electroanalytical Chemistry, 2002, 528, 114-120.

128. V. Viswanathan, H. A. Hansen, J. Rossmeisl, T. F. Jaramillo, H. Pitsch and J. K. Nørskov, The Journal of Physical Chemistry C, 2012, 116, 4698-4704.

129. V. R. Stamenkovic, B. Fowler, B. S. Mun, G. Wang, P. N. Ross, C. A. Lucas and N. M. Marković, Science, 2007, 315, 493-497.

130. L. J. G. A. Mitterdorfer, Solid State lonics, 1999, 117, 187-202.

131. L. J. G. A. Mitterdorfer, Solid State lonics, 1999, 117, 203-217.

132. L. J. G. A Bieberle, Solid State Ionics, 2002, 146, $23-$ 41.

133. S. V. Puranik, A. Keyhani and F. Khorrami, Energy Conversion, IEEE Transactions on, 2010, 25, 804813.

134. F. Grasser and A. C. Rufer, 2007.

135. K. S. Myungsoo Jun, Peter Graf, Journal of Power Sources, 2015, 273, 1226-1236.

136. X. Zhou, E. J. M. Hensen, R. A. van Santen and C. Li, Chemistry - A European Journal, 2014, 20, 69156926.

137. M. A. Henderson, The Journal of Physical Chemistry C, 2015, 119, 19976-19986.

138. O. Neufeld and M. C. Toroker, The Journal of Physical Chemistry C, 2015, 119, 5836-5847.

139. P. Liao, J. A. Keith and E. A. Carter, Journal of the American Chemical Society, 2012, 134, 1329613309.

140. M. J. Janik, C. D. Taylor and M. Neurock, Journal of The Electrochemical Society, 2009, 156, B126-B135.

141. H. A. Hansen, J. Rossmeisl and J. K. Norskov, Physical Chemistry Chemical Physics, 2008, 10, 3722-3730.

142. W.-N. Zhao and Z.-P. Liu, Chemical Science, 2014, 5, 2256-2264.

143. J.-S. Filhol and M. Neurock, Angewandte Chemie International Edition, 2006, 45, 402-406. 